Article

\title{
Genome-Wide Analysis of Cotton Auxin Early Response Gene Families and Their Roles in Somatic Embryogenesis
}

\author{
Ruibin Sun ${ }^{1}$, Shaohui Wang ${ }^{1}$, Dan Ma ${ }^{1}$, Yilin Li $^{2}$ and Chuanliang Liu ${ }^{1,2, *(D)}$ \\ 1 State Key Laboratory of Cotton Biology, Institute of Cotton Research, Chinese Academy of Agricultural \\ Sciences, Anyang 455000, China; sunruibin@caas.cn (R.S.); wangshaohui@caas.cn (S.W.); \\ madan@caas.cn (D.M.) \\ 2 Zhengzhou Research Base, State Key Laboratory of Cotton Biology, Zhengzhou University, \\ Zhengzhou 450001, China; 16638246238@163.com \\ * Correspondence: liuchuanliang@caas.cn
}

Received: 14 August 2019; Accepted: 19 September 2019; Published: 20 September 2019

\begin{abstract}
Auxin is well known to regulate growth and development processes. Auxin early response genes serve as a critical component of auxin signaling and mediate auxin regulation of diverse physiological processes. In the present study, a genome-wide identification and comprehensive analysis of auxin early response genes were conducted in upland cotton. A total of 71 auxin response factor (ARF), 86 Auxin/Indole-3-Acetic Acid (Aux/IAA), 63 Gretchen Hagen3 (GH3), and 194 small auxin upregulated RNA (SAUR) genes were identified in upland cotton, respectively. Phylogenetic analysis revealed that the $A R F$, GH3, and SAUR families were likely subject to extensive evolutionary divergence between Arabidopsis and upland cotton, while the $A u x / I A A$ family was evolutionary conserved. Expression profiles showed that the $A R F, A u x / I A A, G H 3$, and SAUR family genes were extensively involved in embryogenic competence acquisition of upland cotton callus. The $A u x / I A A$ family genes generally showed a higher expression level in the non-embryogenic callus (NEC) of highly embryogenic cultivar CCRI24 than that of recalcitrant cultivar CCRI12, which may be conducive to initializing the embryogenic transformation. Auxin early response genes were tightly co-expressed with most of the known somatic embryogenesis (SE) related genes, indicating that these genes may regulate upland cotton SE by interacting with auxin early response genes.
\end{abstract}

Keywords: Gossypium hirsutum; somatic embryogenesis; ARF; Aux/IAA; GH3; SAUR

\section{Introduction}

Upland cotton (Gossypium hirsutum) is an important economic crop, produces large amounts of natural fibers as raw material for the textile industry, and is used to extract nutrient-rich edible oil from cottonseed [1,2]. Given its substantial economic worth, the improvement of yield, fiber quality, stress tolerance, disease resistance, and other desired traits of cotton are important. Genetic improvement of cotton by transgenesis or genome editing technology is a mainstay for cotton breeding to widen the germplasm genetic diversity and generate transgenic cotton varieties with improved agronomic traits [3]. In cotton, Agrobacterium-mediated genetic transformation has been the most widely used approach to incorporate exogenous genes into explants [4,5]. However, this transformation method is genotype-dependent, and upland cotton with different genotypes has a variable somatic embryogenesis ability during regeneration. Although a relatively effective regeneration system has been established in several upland cotton cultivars, most of the desirable cultivars with elite traits remain difficult to regenerate $[4,6,7]$. The inability of regeneration via somatic embryogenesis severely constrains cotton genetic engineering. 
Somatic embryogenesis is a complicated multi-step process whereby the transformed explant cells/tissues dedifferentiate into callus, then acquire the embryogenic potency to develop into a somatic embryo, which ultimately grows to be a plantlet $[5,8]$. The acquirement of embryogenic potential, viz the transition from non-embryogenic callus to embryogenic callus, is very crucial for somatic embryogenesis and has been suggested as the key bottleneck step for the regeneration of cotton $[9,10]$. Phytohormones have been found to be extensively involved in somatic embryogenesis in many plant species [11], of which auxin and cytokinin have been suggested to be the most important factors affecting the acquisition of embryogenic potential. In cotton, endogenous auxin was significantly increased in embryogenic callus when compared to non-embryogenic callus, and large numbers of auxin signal transduction related transcripts were identified during the transformation from non-embryogenic callus (NEC) to embryogenic callus (EC) [12-14]. This suggests that auxin signaling and response might play important roles in acquiring the embryogenic competence of cotton callus.

Auxin response factors (ARFs), Auxin/Indole-3-Acetic Acids (Aux/IAAs), Gretchen Hagen3s (GH3s), and small auxin upregulated RNAs (SAURs) have been identified as major early auxin responsive genes, serve as critical component of auxin signaling and homeostasis, regulate downstream auxin late response genes in the transcriptional network, and further mediate auxin regulated diverse physiological processes [15-17]. ARF genes encode a class of auxin response transcription factors, which contains several described functional domains: a plant specific B3-type DNA binding domain (DBD) at the amino-terminal, a transcriptional activation or repression domain in the middle region (MR), and a carbon-terminal dimerization domain (CTD) in most cases [18]. DBD makes the ARFs specifically bind to the auxin response cis-element $(A u x R E)$ in the promoters of auxin response genes such as GH3S and SAURs. The MR domain functions to activate or repress the expression of targeted genes when ARFs are recruited by AuxRE. CTD enables ARFs to dimerize with other ARFs or interact with heterologous co-regulation factors such as Aux/IAA [16,18]. The Aux/IAA family genes encode short-lived transcriptional regulator proteins that contain four conserved domains referred to as domain I to IV. The domain III and domain IV sequences are highly homologous with the CTD regions of ARFs and contribute to the interaction of Aux/IAA and ARF [19]. In auxin regulated gene expression, Aux/IAA acts as a repressor of the transcription of auxin response genes by recruiting associated corepressors to interact with ARF, resulting in the repression of the activation activity of ARF [20]. While Aux/IAA protein homeostasis is auxin dependent, Aux/IAA can interact with the auxin receptor protein TRANSPORT INHIBITOR RESPONSE1 (TIR1) and form the TIR1-Aux/IAA complex. When high levels of auxin are perceived by TIR1, the formation of the auxin-TIR1-Aux/IAA complex triggers the degradation of the Aux/IAA repressor, which derepressed the activity of ARF and enabled the activation of targeted auxin response genes [16,21]. Thus, the Aux/IAA interacted CTD of the ARF confers the expression regulation of auxin inducibility of ARFs. Through the mechanism based on Aux/IAA-ARF interaction, ARFs and Aux/IAAs play important roles in regulating the expression of diverse auxin response genes. The GH3 family genes participate in the maintenance of auxin, SA, and JA homeostasis by conjugating excess indole-3-acetic acid to amino acids [16,22], and serves as an essential constituent of the auxin regulation network. SAUR genes comprise a large multigene family that encode small auxin-induced RNAs, and the transcription of SAUR genes can be significantly and sharply induced within $2-5$ minutes during auxin signaling $[23,24]$. SAUR proteins act as key effectors of hormone signals in regulating plant growth and development [25]. In cotton somatic embryogenesis, auxin plays a critical role in a complex manner. ARF and Aux/IAA genes were found differentially expressed during the initial differentiation of somatic embryogenesis in different upland cotton cultivars [26]. Endogenous indoleacetic acid (IAA) content fluctuates in different stages of somatic embryogenesis and surges in embryogenic callus, followed by numerous auxin responsive genes including $A R F, A u x / I A A, G H 3$, and SAUR genes differentially expressed during this developmental process $[13,27]$. Similar results were observed in other studies. Xu et al. (2013) found that endogenous IAA concentration significantly increased during embryogenic competence acquisition in upland cotton somatic embryogenesis, with a number of auxin biosynthesis and signal 
transduction related genes differentially expressed between non-embryogenic callus and embryogenic callus [12]. Furthermore, auxin early response genes including $A R F, A u x / I A A, G H 3$, and SAUR family members showed significant differential expression between sibling cotton lines with distinct different somatic embryogenesis ability, with some auxin response genes even showing specific expression in a highly embryogenic line [12]. All of these suggest the underlying important roles of auxin response genes in somatic embryogenesis.

As previous studies have indicated, auxin response genes are closely implicated in the acquisition of embryogenic competence in cotton somatic embryogenesis [12,28]. We conducted a genome-wide identification and comprehensive analysis of auxin early response genes in cotton, and explored their regulatory roles and mechanisms in the key step of embryogenic competence acquisition in cotton somatic embryogenesis. Our study provides valuable insights into the auxin signaling profile and regulatory roles in the cotton somatic embryogenesis.

\section{Materials and Methods}

\subsection{Identification of $A R F, A u x / I A A, G H 3$, and SAUR Genes in Upland Cotton}

Newly assembled genomic data and gene annotation of G. hirsutum was downloaded from public website (http://cotton.hzau.edu.cn/EN/download.php) as described in the corresponding published paper [29]. To identify the gene family members, the hidden Markov models (HMMs) of ARF (PF06507), Aux/IAA (PF02309), GH3 (PF03321), and SAUR (PF02519) were downloaded from the Pfam database (http://pfam.xfam.org/) [30], which then served as queries for the homologues search $(E$-value $<1)$ against the proteome of G. hirsutum conducted by HMMER v.3.1b2 software [31]. In addition, we performed a similarity search (E-value $<1 \times 10^{-5}$, identity $>50 \%$ ) against the proteome of G. hirsutum by BLAST+ v.2.6.0 [32] with the amino acid sequences of previously reported Arabidopsis ARFs, Aux/IAAs, GH3s, and SAURs as queries. The sum total resulting protein hits from the HMMER search and BLAST search were used for subsequent filtering. Furthermore, InterProScan v.5.32-71.0 [33] was used as the domain prediction to test the presence of characteristic domains. Items containing "Auxin response factor" domain (IPR010525) were recognized as ARFs; items containing the "Aux/IAA" domain (IPR033389) or "PB1" domain (IPR000270), but no "Auxin response factor" domain were recognized as Aux/IAAs; items containing the "GH3" domain (IPR004993) were recognized as GH3s; and items containing the "Small auxin-up RNA" domain (IPR003676) were recognized as SAURs.

\subsection{Phylogenetic Analysis and Gene Structure of Upland Cotton ARF, Aux/IAA, GH3, and SAUR Genes}

Amino acid sequences of $A R F, A u x / I A A, G H 3$, and SAUR family genes identified in G. hirsutum and previous reported Arabidopsis members were combined to perform multiple sequences alignment by MUSCLE [34]. The phylogenetic tree was constructed by IQTREE [35] using the maximum likelihood (ML) statistical method and the best substitution model selected automatically.

Coordinates of the exon-intron and characteristic domains of $A R F, A u x / I A A, G H 3$, SAUR genes were extracted from the genomic gene annotation and InterProScan annotation results, respectively. TBtools was used to display the gene structure and characteristic domain coding regions.

2.3. Genomic Distribution, Collinearity and Duplication Analysis of Upland Cotton ARF, Aux/IAA, GH3, and SAUR Genes

Based on the genomic coordinates of the G. hirsutum ARF, Aux/IAA, GH3, and SAUR genes retrieved from genomic gene annotation, the R package RIdeogram [36] was used to map genes on the chromosomes. MCScanX [37] was used to identify collinearity blocks in G. hirsutum. Collinearity relations of $A R F, A u x / I A A, G H 3$, and $S A U R$ genes were displayed by the circular figure plotted by Circos v.0.69 [38]. Genes in the same family were recognized as derived from segmental duplication when located in collinear blocks. Tandem duplications were detected using MCScanX with the default parameters. 
2.4. Expression Profile Analysis of ARF, Aux/IAA, GH3, and SAUR Genes during Embryogenic Ability Acquisition of Upland Cotton Callus

We previously conducted RNA sequencing of the key step of cotton somatic embryogenesis, where non-embryogenic callus (NEC) and embryogenic callus (EC) from two upland cotton cultivars with different somatic embryogenesis ability (recalcitrant cultivar CCRI12 and highly embryogenic cultivar CCRI24) were sampled (NEC and EC forming CCRI12 are referred to as AN and AE, respectively, NEC and EC forming CCRI24 are referred as BN and BE, respectively) and sequenced using the Illumina platform [28]. This enabled us to investigate the expression profiles of auxin early response genes during cotton somatic embryogenesis. By mapping the sequencing reads to the newly assembled G. hirsutum genome as a reference using Hisat 2 [39], StringTie [40] was used to conduct the quantification of genes and transcripts. Transcripts per million (TPM) read values were computed to represent the gene expression levels. Heatmaps with hierarchy clustering were plotted based on log 10-transformed TPM values using the $\mathrm{R}$ software package pheatmap to visualize the gene expression profiles.

\subsection{Differentially Expression Analysis}

We identified differentially expressed genes (DEGs) for a comparison of different tissue types and cultivars. DESeq 2 [41] was used for different ially expressed gene (DEG) identification based on the read count matrix derived from the StringTie results. Genes showing an absolute value of expression level fold change (FC, represents the ratio of expression level as the TPM value between different samples in a comparison pair) $>2$ and q-value $<0.05$ were recognized as DEGs. Over-representation analysis by the hypergeometric test method was conducted using the $\mathrm{R}$ package clusterProfiler v.3.6.0 [42] based on all of the identified DEGs. To avoid the defects of a hypergeometric test that only focus on genes with a large fold change, the gene set enrichment analysis (GSEA) was conducted based on all expressed genes. Each $A R F, A u x / I A A, G H 3$, and SAUR gene family was defined as a gene set. The $p$-value resulting from DESeq 2 was preprocessed to be transformed into a metric for each gene. Resulted metrics were used as the input of GSEA by running the GSEAPreanked tools in the Broad Institute's GSEA software [43].

To further compare the expression patterns of differentially expressed $A R F, A u x / I A A, G H 3$, and $S A U R$ genes during callus embryogenic potential acquisition, heatmaps were plotted based on the log 2-transformed gene TPM values.

\subsection{Co-Expression Network Analysis}

Based on the expression profiles of all the differentially expressed genes during upland cotton somatic embryogenesis, we computed the Pearson's correlation coefficients between gene pairs using R. Genes with correlation coefficients $>0.98$ were recognized as co-expressed. Co-expression between auxin early response genes and somatic embryogenesis related genes were focused and a co-expression network was constructed and displayed by Cytoscape v.3.7.1 [44].

\section{7. qRT-PCR Validation and Analysis}

qRT-PCR was conducted to validate the expression profiles of auxin early response genes derived from RNA sequencing. Twelve representative genes including two $A R F$, six $A u x / I A A$, two GH3, and two SAUR genes were selected for qRT-PCR validation. Specific primers were designed using the National Center for Biotechnology Information (NCBI) Primer-BLAST (https:/www.ncbi.nlm.nih.gov/ tools/primer-blast/index.cgi) and synthesized commercially (Sangon Biotech). cDNA was synthesized from RNA samples of NEC and EC of CCRI12 and CCRI24 using a HiScript III 1st Strand cDNA Synthesis Kit (Vazyme Biotech Co. Ltd, Nanjing, China) according to the manufacturer's instructions. The obtained cDNA products were diluted five times prior to amplification. qPCR was conducted with a $20 \mu \mathrm{L}$ reaction system containing $1 \mu \mathrm{L}$ cDNA templates, $10 \mu \mathrm{L} 2 \times$ TransStart Top Green qPCR Super Mix (TransGen Biotech Co. Ltd, Beijing, China), $0.5 \mu \mathrm{L}$ of each $10 \mu \mathrm{M}$ forward and reverse primers, and 
$8 \mu \mathrm{L} \mathrm{ddH_{2 }}$ O. All qPCR reactions were performed in triplicate on a Roche Light Cycler 480 instrument. A thermal cycling program was performed with pre-incubation at $95^{\circ} \mathrm{C}$ for $5 \mathrm{~min}$, followed by 40 cycles of denaturation at $95^{\circ} \mathrm{C}$ for $10 \mathrm{~s}$, annealing at $58^{\circ} \mathrm{C}$ for $10 \mathrm{~s}$ and extension at $72{ }^{\circ} \mathrm{C}$ for $10 \mathrm{~s}$. Relative quantitation of gene expression was computed using the online tool A shiny for the analysis of real-time PCR data (https://ihope.shinyapps.io/qRT-PCR-Pipeline/) with GhHis3 (GenBank: AF024716) was used as the internal reference.

\section{Results}

\subsection{Identification and Characterization of ARF, Aux/IAA, GH3, and SAUR Genes in Upland Cotton}

A total of $71 A R F, 86 A u x / I A A, 63$ GH3, and 194 SAUR genes were identified in upland cotton. All of the identified upland cotton ARFs have a B3-type DBD, ARF domain, and Aux/IAA-like CTD at the same time. Of these, $24 \mathrm{ARFs}$ whose $\mathrm{MR}$ were enriched in glutamine $(\mathrm{Q})$, serine $(\mathrm{S})$, and leucine $(\mathrm{L})$ were considered as activators, while other ARFs rich in serine (S), proline (P), and leucine (L) were probable transcriptional repressors $[18,45]$ (Table 1, Supplementary Figure S1). Most of the identified upland cotton Aux/IAAs harbored all of the typical four conserved domains (Domains I-IV), while some of the Aux/IAAs in upland cotton were truncated. Sixteen Aux/IAAs lacked the "LxLxL" motif-containing Domain I, which has the function of ethylene response factor-associated amphiphilic repression. Two Aux/IAAs lacked Domains I and II at the same time. Two and three Aux/IAAs lacked Domains III and IV, respectively, one Aux/IAA lacked Domains III and IV at the same time, while the other lacked Domains III and II at the same time (Table 1). The canonical degron motif "GWPPV", which confers rapid auxin-induced Aux/IAA degradation, existed in the Domain II of upland cotton Aux/IAAs [19,46]. Additionally, the previously reported rate motif "KR" located between Domains I and II is present in upland cotton Aux/IAAs and controls the auxin-induced degradation rate of Aux/IAAs [47] (Supplementary Figure S2). All identified upland cotton GH3s had a conserved GH3 domain; within the eight GH3s contained $2 \times$ GH3 domains, while the other eight GH3s contained the Jasmonic acid-amino synthetase (JAR1) domain simultaneously (Table 1). Previous study showed that some conserved motifs/residues are necessary for GH3s' hormone-amino synthetase activity including four hormone-binding motifs/residues and three ATP/AMP-binding motifs/residues [48]. In the present study, almost all identified upland cotton GH3s contained all of these functional motifs/residues, except for three C-terminal truncated GH3s (Ghir_A04G003360.1, Ghir_A01G005350.1, Ghir_A01G008830.1) and two N-terminal truncated GH3s (Ghir_D04G015220.1, Ghir_A11G031180.1) lacking some of functional motifs/residues, and in particular, a severely truncated GH3 (Ghir_A11G031160.1) contained only one ATP/AMP-binding motif/residue (Supplementary Figure S3). All identified upland cotton SAURs contained a SAUR domain and auxin-inducible motif (Table 1, Supplementary Figure S4). The identification results of the auxin early response genes in the present study was almost consistent with previous studies. The number of G. hirsutum ARF genes (71) identified in the present study was almost the sum of the number of $A R F$ genes in its diploid ancestor species as each G. arboreum and G. raimondii contained 35 ARF genes $[49,50]$. Previous study identified 56 GH3 genes in G. hirsutum (NAU-NBI, v1.1) [48,51], while 63 were identified in the present study. Nevertheless, the present study identified the same number of GH3 subfamily II gene members as the previous study [48]. The present study identified fewer SAUR genes than the previous study [52]. In a previous study, more than twenty of the identified SAUR genes were located on scaffolds, while all 194 SAUR genes identified in the present study were located on chromosomes [52]. The difference in the identification results may be due to the different genome assembly and identification methods adopted. We supposed that some scaffolds may be assembled onto chromosomes in the new genome assembly versus the one before, making a more accurate identification of the gene members. 
Table 1. Characteristics of identified auxin response factor (ARF), Auxin/Indole-3-Acetic Acid (Aux/IAA), Gretchen Hagen3 (GH3), and small auxin upregulated RNA (SAUR) genes in upland cotton.

\begin{tabular}{|c|c|c|}
\hline Gene Family & Domains/Motifs & Number of Genes \\
\hline \multirow[t]{3}{*}{$\mathrm{ARF}$} & & 71 \\
\hline & DBD, ARF-AD ${ }^{\mathrm{a}}, \mathrm{Aux} / \mathrm{IAA}$ & 24 \\
\hline & DBD, ARF-RD ${ }^{a}, A u x / I A A$ & 47 \\
\hline \multirow[t]{8}{*}{ Aux/IAA } & & 86 \\
\hline & Domains I, II, III, IV & 67 \\
\hline & Domains I, II, III & 1 \\
\hline & Domains II, III, IV & 13 \\
\hline & Domains I, II & 1 \\
\hline & Domains I, IV & 1 \\
\hline & Domains II, III & 1 \\
\hline & Domains III, IV & 2 \\
\hline \multirow[t]{4}{*}{ GH3 } & & 63 \\
\hline & GH3 & 47 \\
\hline & $2 \times \mathrm{GH} 3$ & 8 \\
\hline & GH3, JAR1 & 8 \\
\hline \multirow[t]{7}{*}{ SAUR } & & 194 \\
\hline & SAUR, Motifs 1 b , 2, 3 & 167 \\
\hline & SAUR, Motifs 1, 2 & 9 \\
\hline & SAUR, Motifs 1, 3 & 2 \\
\hline & SAUR, Motifs 2, 3 & 12 \\
\hline & SAUR, Motif 2 & 3 \\
\hline & SAUR, Motif 3 & 1 \\
\hline
\end{tabular}

\subsection{Phylogenetic Analysis and Gene Structure of ARF, Aux/IAA, GH3, and SAUR Genes in Upland Cotton}

We constructed phylogenetic trees by the maximum likelihood (ML) method based on full length amino acid sequences of identified ARF, Aux/IAA, GH3, and SAUR protein in upland cotton with corresponding gene family members in Arabidopsis as a reference (Figure 1). Results showed that most clades had robust bootstrap support, and the topological classification of Arabidopsis genes in our ML trees were consistent with previous studies. According to the ML tree, ARF genes were classified into six distinct groups. Group I contained genes only from Arabidopsis, while all of the other groups were shared by Arabidopsis and upland cotton genes (Figure 1a). All transcriptional activators of ARFs were distributed in Groups V and VI. The $A u x / I A A$ genes were classified into ten groups, all groups were shared by Arabidopsis and upland cotton (Figure 1b). GH3 genes were divided into six groups, except for Group I where all members belonged to Arabidopsis, and Group II where all members belonged to upland cotton; all other groups were shared with Arabidopsis and upland cotton (Figure 1c). The SAUR genes were divided into eight groups, almost all groups were shared by Arabidopsis and upland cotton, except for Group I, which consisted of only Arabidopsis gene members (Figure 1d). These results suggest that the $A u x / I A A$ family genes are relatively conserved between Arabidopsis and upland cotton during evolution, while the $A R F, G H 3$, and $S A U R$ family genes may experience more extensive evolutionary divergence as some clades exhibit species specificity.

Gene exon-intron structure analysis showed that genes in each clade exhibited comparable exon-intron structure between Arabidopsis and upland cotton (Supplementary Figure S5), implying the evolutionary conservation of these gene members between Arabidopsis and upland cotton. Gene exon-intron structure and intron number maintained relatively stable within groups, while it varied between different groups in the $A R F, A u x / I A A$, and GH3 families, which almost coincided with the classification in the ML trees. The $A R F$ genes had the most introns (average $10.38 \pm 4.35$ ), while the $A u x / I A A$ and $G H 3$ genes had less introns (average $4.52 \pm 1.10$ and $3.90 \pm 0.86$, respectively). Interestingly, the majority of upland cotton $A R F$ genes had 10 to 15 introns, while the ARF gene members in Group IV 
contained significantly less introns, which varied from 2 to 4 . The majority of SAUR genes had no introns, and this could be attributed to the short length of SAUR genes (most SAUR genes are less than $1 \mathrm{~kb}$ ).

a
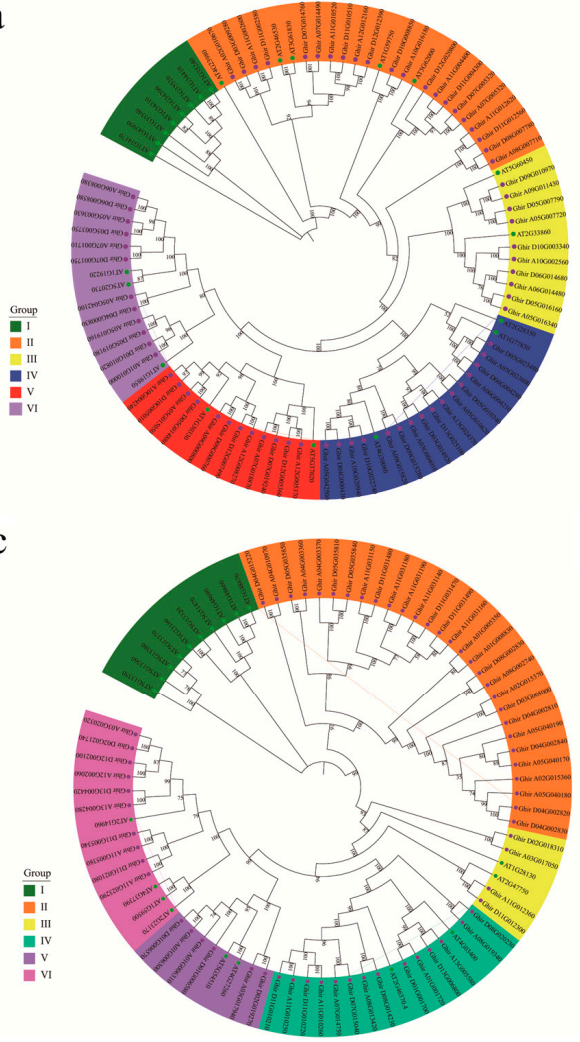

b

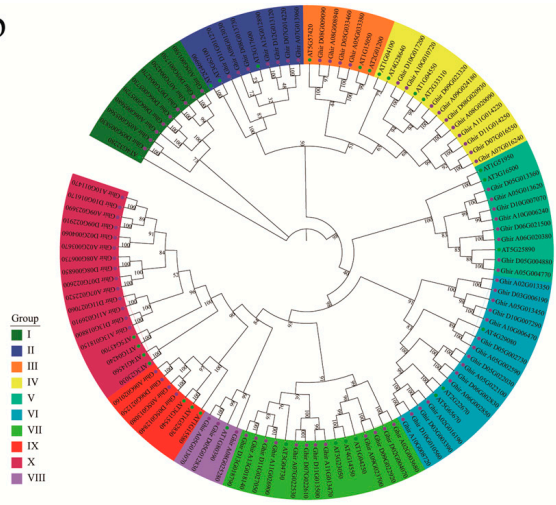

d

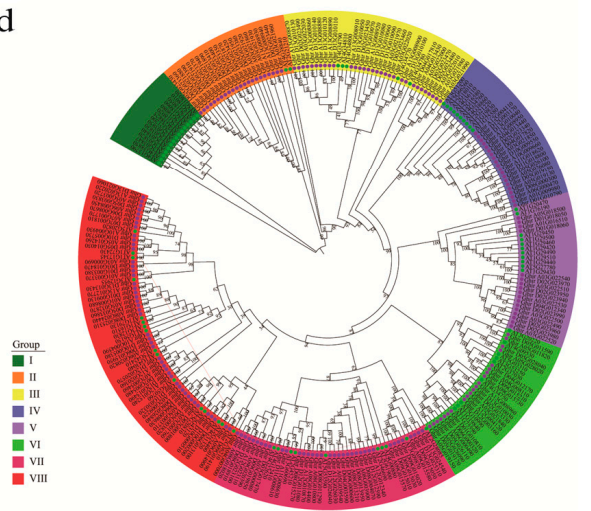

Figure 1. Phylogenetic trees of $A R F(\mathbf{a}), A u x / I A A(\mathbf{b}), G H 3(\mathbf{c})$, and SAUR (d) family genes from Arabidopsis and upland cotton. The trees were constructed by the maximum likelihood (ML) method based on the full length amino acid sequence of the ARF, Aux/IAA, GH3, and SAUR proteins.

\subsection{Genomic Distribution and Gene Duplication of ARF, Aux/IAA, GH3, and SAUR Genes in Upland Cotton}

Based on the genes coordinate annotation data, we mapped identified upland cotton $A R F$, $A u x / I A A, G H 3$, and SAUR genes on chromosomes (Figure 2a). All of these genes are located on assembled chromosomes and are distributed unevenly across different chromosomes, with none on the scaffolds. All 71 identified $A R F$ genes were distributed on 24 of the total 26 chromosomes; no $A R F$ gene was located on chromosomes A04 and D02. Similarly, 86 Aux/IAA genes were distributed on 21 chromosomes; moreover, there was no $A u x / I A A$ gene on chromosomes A01, A03, A04, D01, and D04. Moreover, 63 GH3 genes were distributed on 20 chromosomes, with no GH3 gene on chromosomes A06, A09, A10, D06, D09, and D10. Additionally, 194 SAUR genes were distributed across all 26 upland cotton chromosomes with dense distributions on the terminal of chromosomes A03 and D02, which was largely similar to a previous study [52]. Based on the results of the collinearity analysis, we found that the majority of $A R F, A u x / I A A, G H 3$, and $S A U R$ genes belonged to segmental duplicates resulting from polyploidy and chromosome rearrangements. There were $59 A R F, 90 A u x / I A A, 38$ GH3, and 187 SAUR homologous gene pairs involving 71 ARF genes, 85 Aux/IAA genes, 55 GH3 genes, and 175 SAUR genes located in segmental duplication blocks, respectively (Figure 2b). Furthermore, one, 10, and 17 gene sets involving two $A u x / I A A$ genes, 23 GH3 genes, and 43 SAUR genes, respectively, were determined as tandem duplication, and no tandem duplication was observed in upland cotton $A R F$ genes (Figure 2a). This implied that segmental duplication extensively existed in the $A R F, A u x / I A A$, $G H 3$, and $S A U R$ gene families, and tandem duplication further gave rise to more gene members of the GH3 and SAUR families. 
a

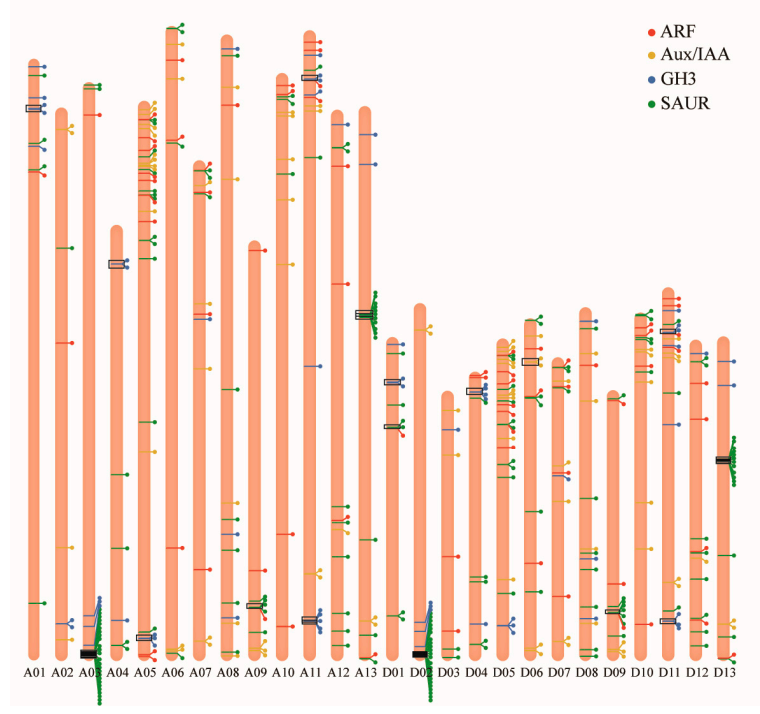

b

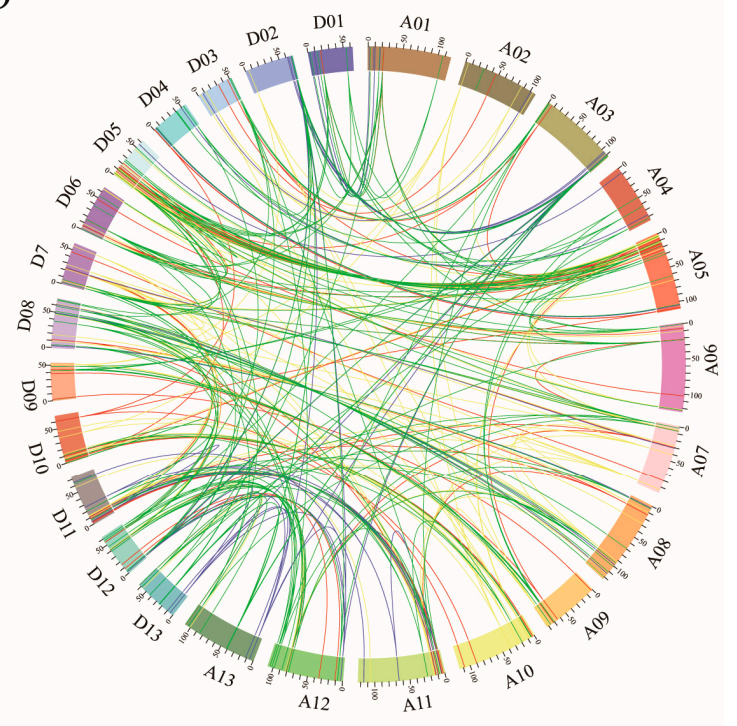

Figure 2. Chromosomal distribution (a) and collinearity (b) of the identified $A R F, A u x / I A A$, GH3, and $S A U R$ genes in upland cotton indicated gene duplications. (a) Genes were displayed by striping on chromosomes, circle points were labeled at the end of stripes to make a clearer distinction between adjacent genes from each other. $A R F, A u x / I A A, G H 3$, and $S A U R$ genes are represented by red, yellow, blue, green stripes and labels, respectively. Tandem duplicate genes were surrounded by black rectangles to represent tandem duplication gene sets. (b) Chromosomal segmental duplication regions were analyzed by MCScanX, ARF, Aux/IAA, GH3, and SAUR homologous gene pairs in segmental duplication regions linked with different color curves (red, yellow, blue, and green, respectively).

3.4. Expression Profiling and Differentially Expression of ARF, Aux/IAA, GH3, and SAUR Genes in Upland Cotton Somatic Embryogenesis

As the auxin signaling pathway was found to play an important role in cotton somatic embryogenesis, we focused on the expression pattern of auxin early response $A R F, A u x / I A A, G H 3$, and SAUR genes during this process. We previously conducted RNA sequencing on the bottleneck step of upland cotton somatic embryogenesis and the acquisition of embryogenic potential in the transformation from non-embryogenic callus (NEC) to embryogenic callus (EC) [28] by using two upland cotton cultivars with different somatic embryogenesis ability (recalcitrant embryogenic cultivar CCRI12 and highly embryogenic cultivar CCRI24). This enabled us to elucidate and compare the transcriptomic dynamics of $A R F, A u x / I A A, G H 3$, and SAUR genes in different cultivars. As a result, the majority of $A R F$ and $A u x / I A A$ genes were actively expressed during the acquisition of embryogenic ability. About $94.4 \%$ (67 out of 71) of $A R F$ genes and $83.7 \%$ (72 out of 86 ) of $A u x / I A A$ genes were expressed (TPM value $>1$ ) during the embryogenic transformation of the callus, and $90.1 \%$ (64 out of 71) of $A R F$ genes and $66.3 \%$ (57 out of 86) of $A u x / I A A$ genes were expressed at relatively high levels with TPM $>5$. Almost half of the GH3 genes (37 out of 63) showed expression (TPM value $>1$ ) in this process, with $27 \mathrm{GH} 3$ s showing a TPM $>5$. While the majority of SAUR genes showed a low level of expression throughout the embryogenic ability acquisition with only $18.0 \%$ (35 out of 194) of SAUR genes actively expressed with a TPM value $>5$ (Figure 3, Table S1). This suggests that ARF and $A u x / I A A$ family genes extensively participate in the auxin signaling mediated embryogenic ability acquisition process during upland cotton somatic embryogenesis, where some of the GH3 family gene members were also involved in this process, while only a few SAUR genes were involved. 
a

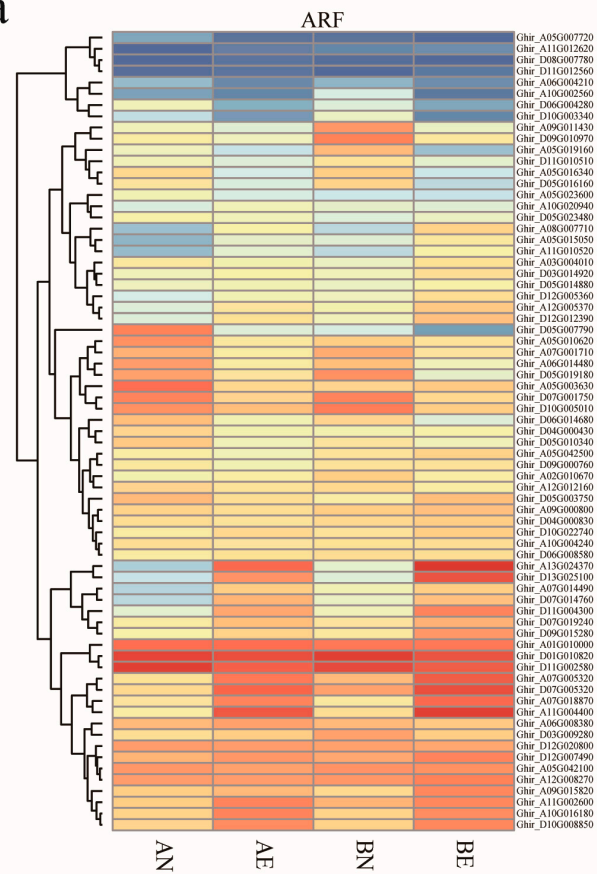

c

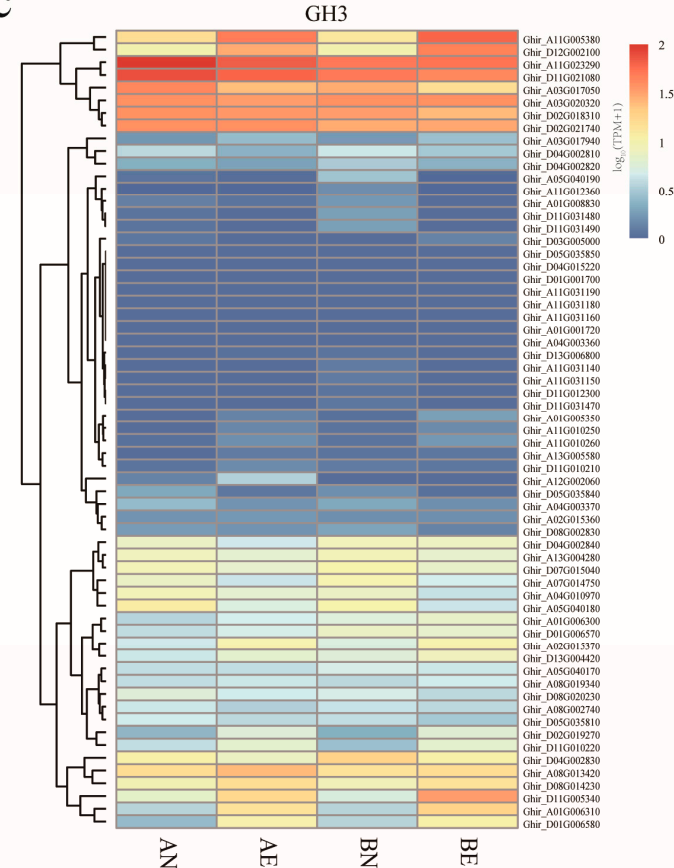

b

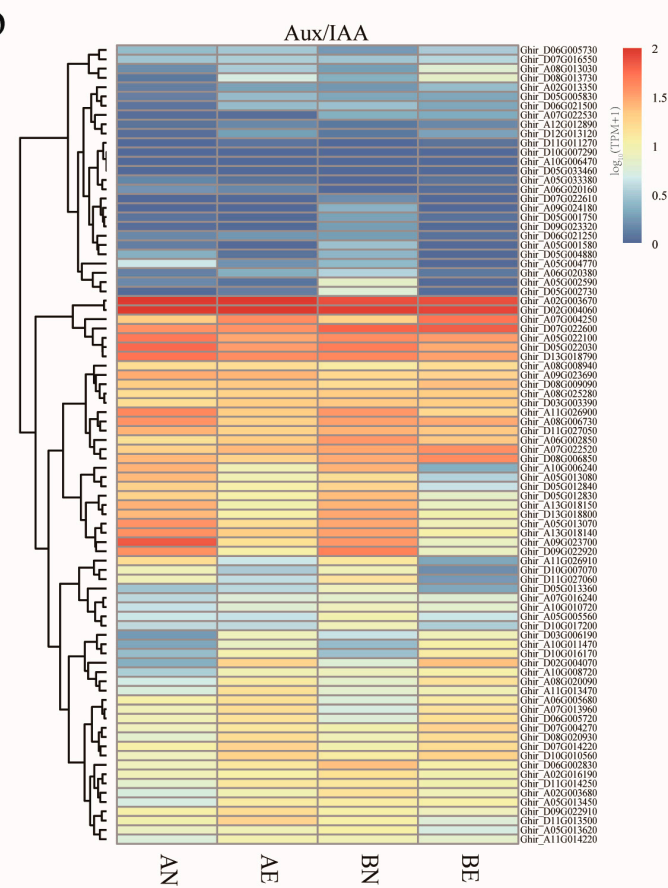

d

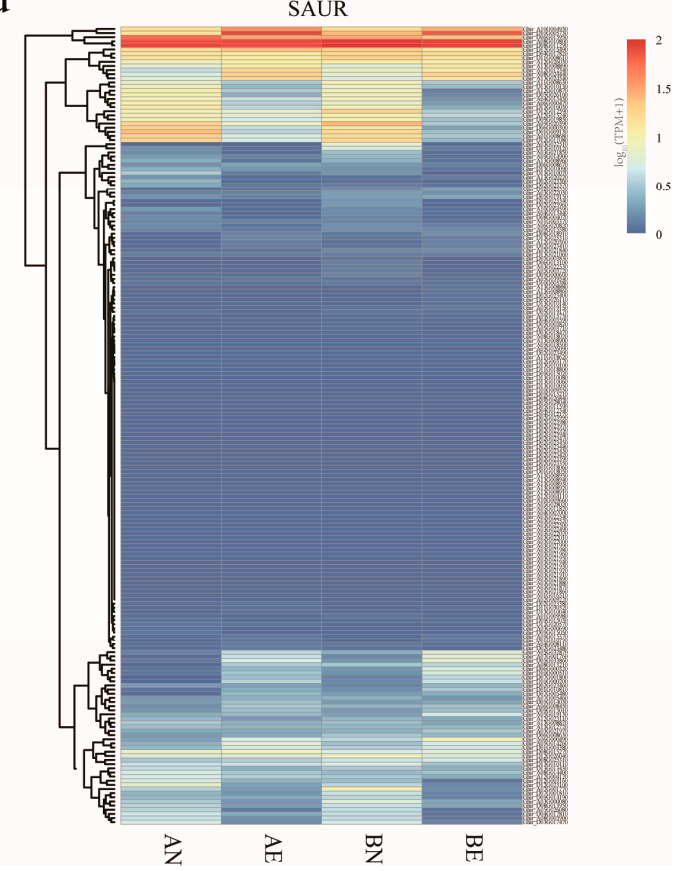

Figure 3. Expression profiles of $A R F(\mathbf{a}), A u x / I A A(\mathbf{b}), G H 3(\mathbf{c})$, and $S A U R$ (d) genes during the acquisition of the embryogenic ability of upland cotton callus from NEC to EC. The heatmaps were drawn based on log 10-transformed TPM values of genes derived from the RNA sequencing analysis. AN and AE represent the NEC and EC of the recalcitrant embryogenic cultivar CCRI12, respectively and BN and BE represent the NEC and EC of highly embryogenic cultivar CCRI24, respectively.

Further analysis found that there were $44 A R F(62.0 \%), 56 A u x / I A A(65.1 \%), 23$ GH3 (36.5\%), and 52 SAUR (26.8\%) genes that were significantly differentially expressed during the process from NEC to EC when using an absolute value of fold change (FC, represents the ratio of expression level as TPM value between different samples in a comparison pair) $>2$ and $q$-value $<0.05$ as the criteria (Figure 4, Table 2). For all four auxin early response gene families, comparisons of AN_vs_AE and 
BN_vs_BE made up the majority of these differentially expressed genes (DEGs), while few auxin early response genes showed significant differentially expression in comparisons AN_vs_BN and AE_vs_BE (Table 2). Twelve differentially expressed auxin early response genes were selected to validate the expression pattern through qRT-PCR (Table S2). The results showed that almost all tested genes showed comparable expression patterns between the qRT-PCR measurements and RNA sequencing analysis, except for one $A u x / I A A$ gene (Figure 5). The qRT-PCR validation results suggest the reliability of RNA sequencing.
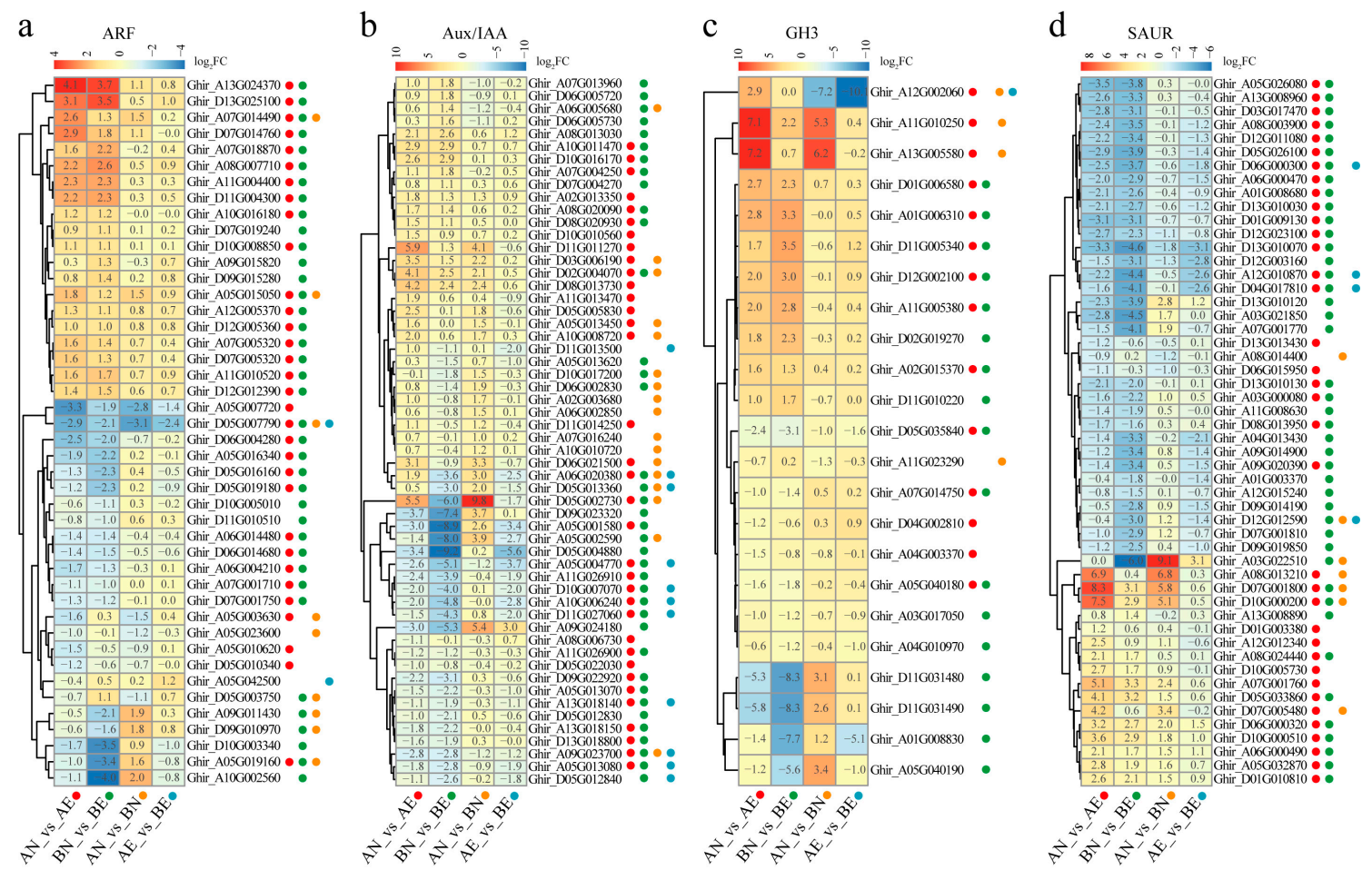

Figure 4. Schematic expression level change of differentially expressed ARF (a), Aux/IAA (b), GH3 (c), and SAUR (d) genes during transformation from NEC to EC. Numbers in grids indicate the log 2-transformed fold change (FC) values. Colored circle points beside the gene names indicate if the corresponding genes were significantly differentially expressed in AN_vs_AE (red), BN_vs_BE (green), AN_vs_BN (orange), and AE_vs_BE (blue) comparison pairs.

Table 2. Statistics of differentially expressed $A R F, A u x / I A A, G H 3$, and $S A U R$ genes during transformation from NEC to EC in upland cotton somatic embryogenesis. Differentially expressed genes (DEGs) were identified in four comparison pairs, respectively.

\begin{tabular}{ccccccc}
\hline DEGs & & AN_vs_AE & BN_vs_BE & AN_vs_BN & AE_vs_BE & Total \\
\hline ARF & & 32 & 38 & 9 & 2 & 44 \\
& up & 17 & 21 & 5 & 1 & - \\
Aux/IAA & down & 15 & 17 & 4 & 1 & - \\
& up & 35 & 38 & 17 & 11 & 56 \\
GH3 & 19 & 12 & 15 & 0 & - \\
& down & 16 & 26 & 2 & 11 & - \\
SAUR & up & 9 & 17 & 4 & 1 & 23 \\
& down & 5 & 9 & 2 & 0 & - \\
& up & 36 & 43 & 7 & 4 & - \\
& down & 21 & 10 & 6 & 0 & - \\
\hline
\end{tabular}




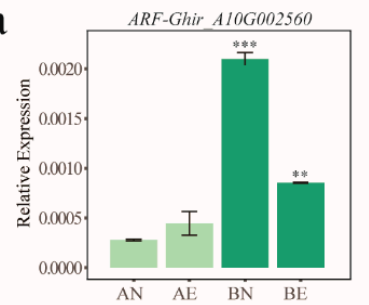

c

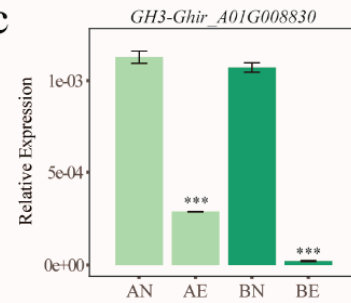

d

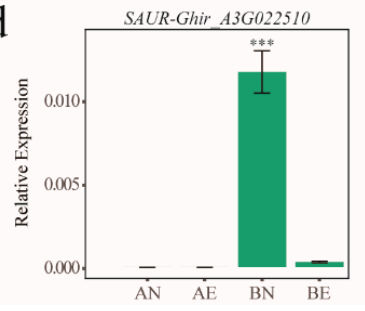

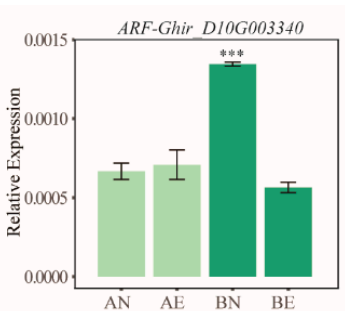
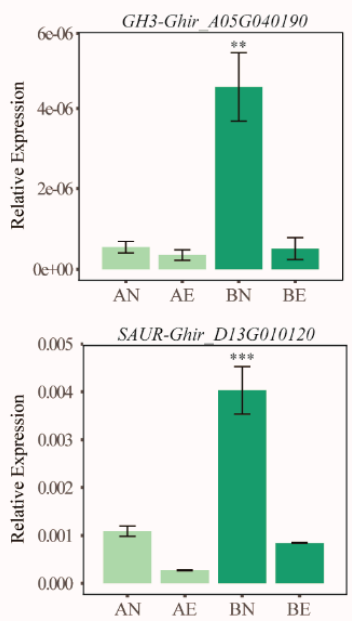

$\mathrm{b}$
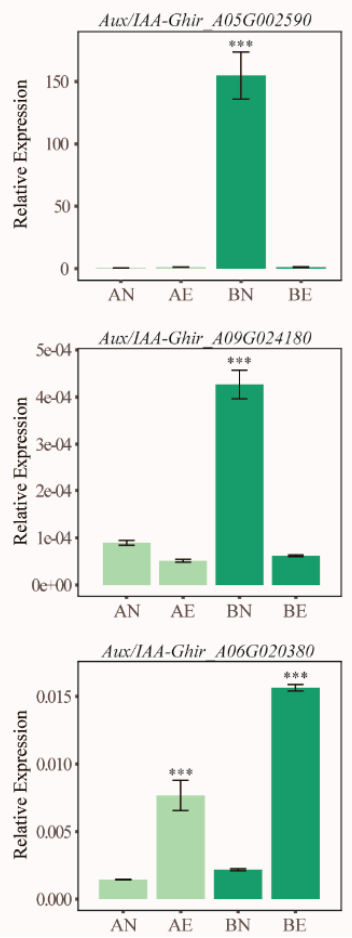
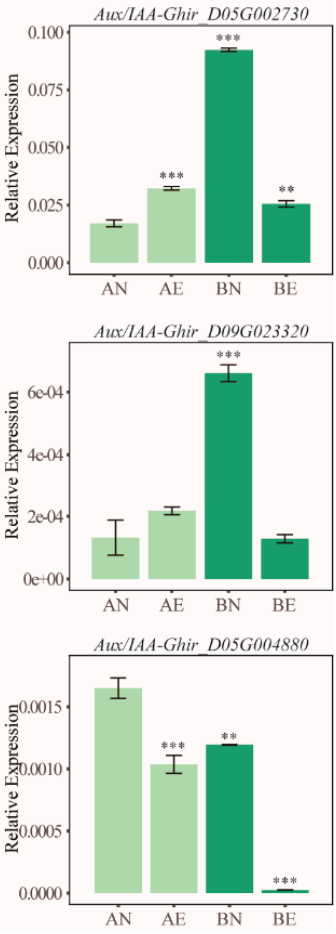

Figure 5. qRT-PCR analysis showing the expression of auxin early response $A R F(\mathbf{a}), A u x / I A A(\mathbf{b}), G H 3$ (c), and SAUR (d) genes during transformation from NEC to EC. Callus samples from CCRI12 and CCRI24 were colored in light green and dark green, respectively. Multiple comparisons of expression levels between different samples were conducted with AN chosen as the reference. Statistically significant differences are displayed with asterisks $\left({ }^{\prime * * * \prime} p\right.$-value $\leq 0.001,{ }^{\prime * * \prime} p$-value $\left.\leq 0.01\right)$.

In most cases, auxin early response genes showed the same expression pattern between the AN_vs_AE and BN_vs_BE comparisons, suggesting that this part of the gene may be critical for callus embryogenic ability acquisition in both the CCRI12 and CCRI24 cultivars (Figure 4). With some exceptions, the Aux/IAA gene Ghir_D05G002730 showed the opposite expression pattern between AN_vs_AE and BN_vs_BE, which was significantly upregulated in CCRI12, but significantly downregulated in CCRI24 during the process from NEC to EC (Figures 4 and 5), implying the transcriptional response difference between different cultivars during embryogenic ability acquisition, which may be implicated in the difference of somatic embryogenesis ability. Moreover, concerning the genes showing the same expression pattern in CCRI12 and CCRI24, most differentially expressed $A R F$ and $G H 3$ genes (70.5\% of $A R F \mathrm{~s}, 60.9 \%$ of $G H 3 \mathrm{~s})$ showed a comparable degree of expression change between AN_vs_AE and BN_vs_BE, of about half of the differentially expressed $A u x / I A A$ and SAUR genes (29/56 (51.8\%) ARFs, 26/52 (50.0\%) GH3s) showed a different degree of expression change (the difference of $\log _{2} \mathrm{FC}$ greater than 1) between AN_vs_AE and BN_vs_BE (Figure 4). For example, the homologous ARF genes Ghir_A10G002560 and Ghir_D10G003340 showed a significant downregulation in CCRI24 during the transformation from NEC to EC, while no significant expression change was found in CCRI12 (Figure 5a). Similar cases were found in the $A u x / I A A$ gene Ghir_A05G002590 and SAUR gene Ghir_A03G022510 (Figure 5b,d). Moreover, for the Aux/IAA genes Ghir_A09G024180, Ghir_D05G004880, and Ghir_A06G020380; the GH3 genes Ghir_A01G008830 and Ghir_A05G040190; and SAUR gene Ghir_D13G010120, they were upregulated or downregulated with a distinct higher ratio in CCRI24 than in CCRI12 (Figure 5).

Over-representation analysis of the hypergeometric test based on all (in both AN_vs_AE, BN_vs_BE, AN_vs_BN, AE_vs_BE comparison pairs) the differentially expressed genes identified in transcriptomic sequencing found that the $A R F$ and $A u x / I A A$ families were significantly over-represented in both CCRI12 and CCRI24 during embryogenic potential acquisition of the callus. Both $A R F$ and 
Aux/IAA families were over-represented in the upregulated DEGs identified in AN_vs_AE, while in BN_vs_BE, only the $A R F$ family was over-represented in upregulated DEGs as the $A u x / I A A$ family was over-represented in downregulated DEGs. In comparison, the AN_vs_BN and $A u x / I A A$ family was enriched in upregulated DEGs (Figure 6a).

Gene set enrichment analysis was conducted based on all genomic gene expression profiles, with the ARF, AuxIAA, GH3, and SAUR families defined as gene sets, respectively. Results showed that in CCRI12, the $A R F$ family gene set was significantly upregulated in AE when compared with AN using the nominal $p$-value $<0.05$ and FDR $q$-value $<0.05$ as criteria. In CCRI24, the $A R F$ family gene set was upregulated in $\mathrm{BE}$ when compared with $\mathrm{BN}$, and the $A u x / I A A$ and $S A U R$ family gene sets were significantly upregulated in $\mathrm{BN}$ when compared with $\mathrm{BE}$. When comparing $\mathrm{AN}$ versus $\mathrm{BN}$, the $A u x / I A A$ family gene set was significantly upregulated in BN when compared with AN (Figure 6b-e). These results were almost consistent with the above over-representation analysis.

a
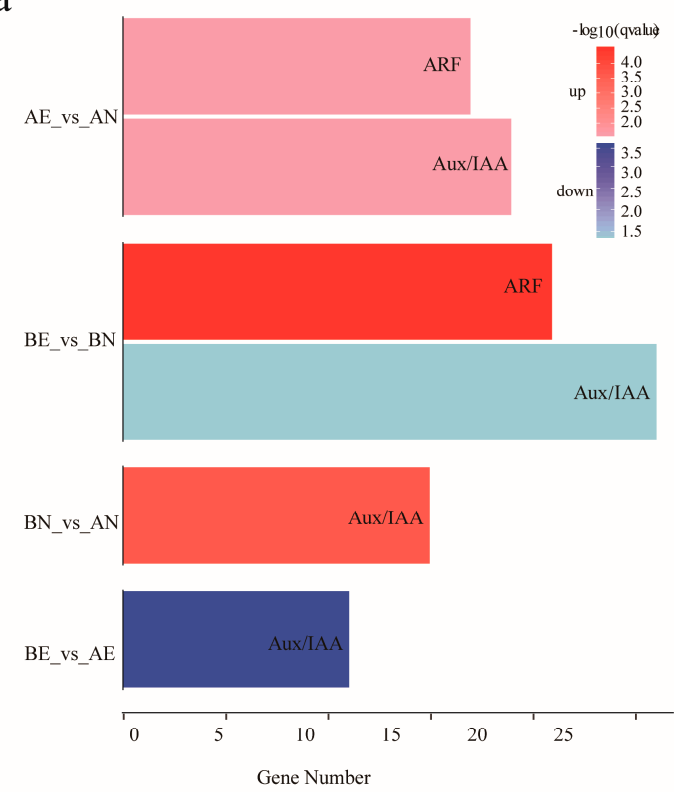

d
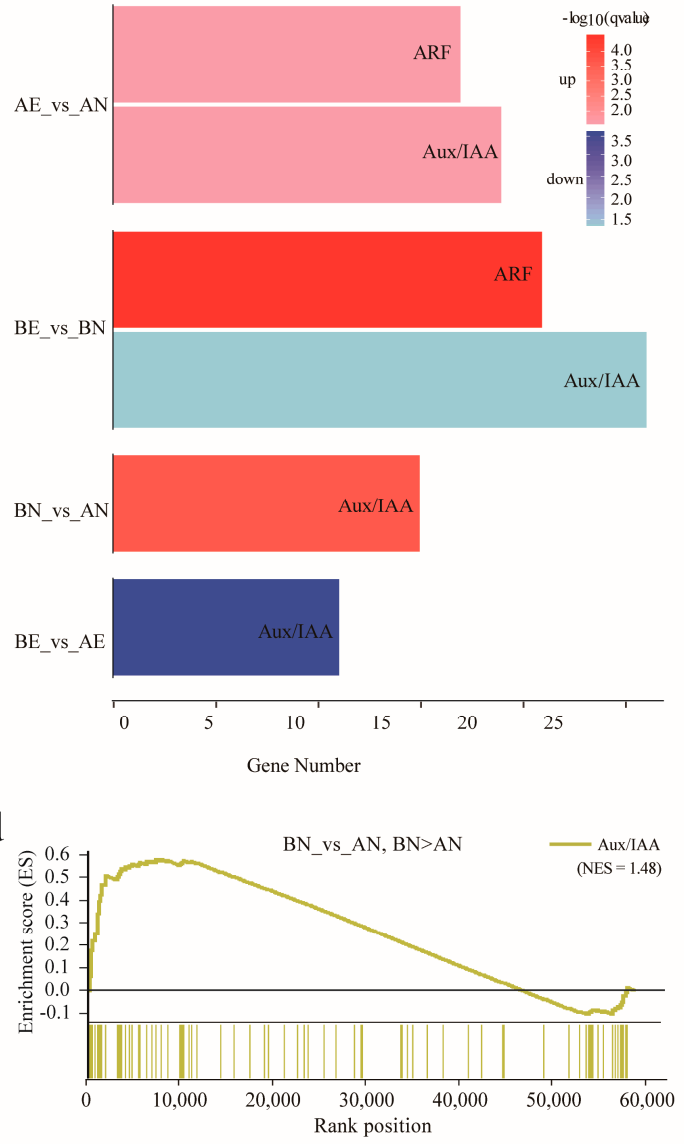

b

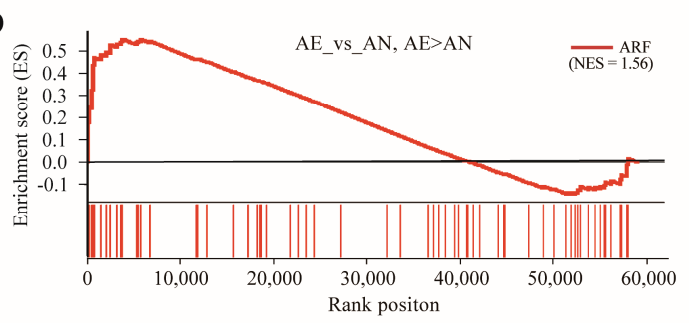

$\mathrm{c}$

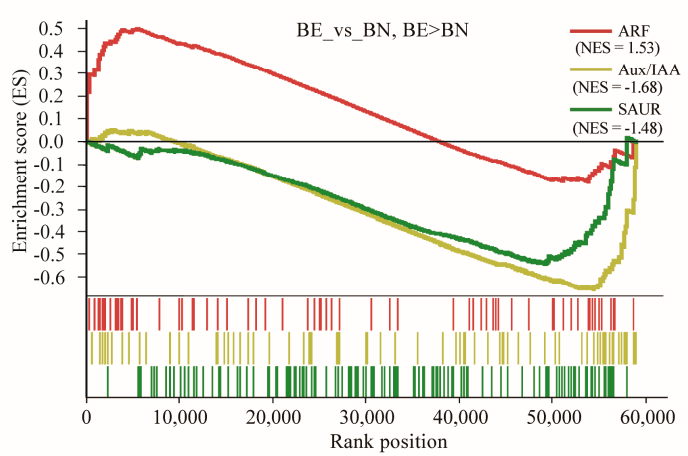

e

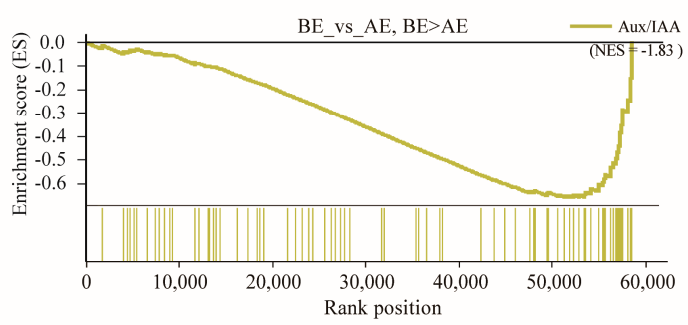

Figure 6. Enrichment results of $A R F, A u x / I A A, G H 3$, and $S A U R$ genes during the acquisition of embryogenic potential. (a) Over-representation analysis plots of the hypergeometric test based on differentially expressed $A R F, A u x / I A A, G H 3$, and SAUR genes. Enriched auxin early response gene families are shown by the colored bars, enriched families in the upregulated and downregulated DEGs are colored by red and blue, respectively. The different shade of the red or blue color indicates the different degree of enrichment represented by the minus of $\log 10$ transformed q values. (b-e) GSEA plots of $A R F, A u x / I A A, G H 3$, and SAUR family gene sets in different comparison pairs, significant enriched family gene sets are shown in the enrichment score (ES) plots by different colors. Normalized enrichment scores (NES) for each enriched family set is shown in brackets. A positive NES value indicates that the family gene set was positively associated with the indicated relativeness in the comparison pair. A negative NES value indicates that the family gene set was negatively associated with the indicated relativeness in the comparison pair. 
3.5. Co-Expression of ARF, Aux/IAA, GH3, and SAUR Genes and Somatic Embryogenesis Related Genes during Cotton Somatic Embryogenesis

Co-expression analysis was conducted among the $A R F, A u x / I A A, G H 3$, and SAUR families. The result showed that the majority of DEGs in all of the four auxin early response families showed a significant expression correlation with each other both within intra- and inter-families (Table 3, Supplementary Figure S6), suggesting that the $A R F, A u x / I A A, G H 3$, and SAUR families are closely collaborated in auxin signaling regulation during upland cotton somatic embryogenesis.

Table 3. Statistics of co-expressed genes among auxin early response families.

\begin{tabular}{cccccc}
\hline \multirow{2}{*}{ Auxin Early Response Family } & \multirow{2}{*}{ DEGs } & \multicolumn{4}{c}{ Number (Percentage) of DEGs Co-Expressed with } \\
\cline { 3 - 5 } & & ARF & Aux/IAA & GH3 & SAUR \\
\hline ARF & 44 & $38(86.4 \%)$ & $39(88.6 \%)$ & $37(84.1 \%)$ & $40(90.9 \%)$ \\
Aux/IAA & 56 & $39(69.6 \%)$ & $41(73.2 \%)$ & $39(69.6 \%)$ & $41(73.2 \%)$ \\
GH3 & 23 & $18(78.3 \%)$ & $19(82.6 \%)$ & $17(73.9 \%)$ & $20(87.0 \%)$ \\
SAUR & 52 & $48(92.3 \%)$ & $49(94.2 \%)$ & $45(86.5 \%)$ & $49(94.2 \%)$ \\
\hline
\end{tabular}

Somatic embryogenesis is a complex developmental process involved in the regulation of many genes and is influenced by various external environment factors including plant hormones. Up to now, a number of genes have been identified to play roles in somatic embryogenesis such as $L E C 1$, LEC2, ABI3, AGL15, FUS3, PLTs, BABY BOOM (BBM), WUSCHEL (WUS), SERKs, and so on [53-60]. To investigate the co-expression relation between auxin early response genes and reported somatic embryogenesis related genes, we constructed co-expression networks of these genes based on their expression profiles during somatic embryogenesis. A total of $26 A R F, 23 A u x / I A A, 12$ GH3, and 29 $S A U R$ genes were tightly correlated with somatic embryogenesis related genes that had Pearson's correlation coefficients $>0.98$ and a p-value $<0.05$ (Table 4, Figure 7). For $A R F, A u x / I A A, G H 3$, and $S A U R$ families, about half of these co-expressed auxin early responsive genes were greatly correlated with somatic embryogenesis related to ABI3, FUS3, PLT2, and PLT5 genes (except that 30.8\% of co-expressed $A R F$ genes were correlated with PLT2). About $40 \%$ of co-expressed auxin early responsive genes were correlated with LEC1 and SERK4, except for ARF family where only 30.8\% co-expressed genes were correlated with SERK4. More than $30 \%$ of co-expressed $A R F, A u x / I A A$, and $S A U R$ genes were correlated with PLT1 and PLT7, as the counterpart percentage in the GH3 family was $25 \%, 50 \%$, and $25 \%$, respectively. Over $20 \%$ of co-expressed auxin early responsive genes were correlated with AGL15 and SERK3 for the ARF, Aux/IAA, GH3, and SAUR families. About $40 \%$ of co-expressed $A u x / I A A$ and $S A U R$ genes were correlated with PLT3, as only $19.2 \%$ and $8.3 \%$ in the $A R F$ and GH3 families, respectively. Few auxin early responsive genes were correlated with $B B M$ and $L E C 2$, however, no gene was correlated with WUS (Table 4).

Table 4. Statistics of the numbers of auxin early response genes co-expressed with somatic embryogenesis related genes.

\begin{tabular}{ccccc}
\hline \multirow{2}{*}{ SE Related Genes } & \multicolumn{4}{c}{ Number (Percentage) of Co-Expressed Auxin Early Response Genes } \\
\cline { 2 - 5 } & ARF & AuxIAA & GH3 & SAUR \\
\hline ABI3 & $14(53.8 \%)$ & $15(65.2 \%)$ & $8(66.7 \%)$ & $18(62.1 \%)$ \\
AGL15 & $8(30.8 \%)$ & $8(34.8 \%)$ & $3(25.0 \%)$ & $9(31.0 \%)$ \\
FUS3 & $12(46.2 \%)$ & $13(56.5 \%)$ & $8(66.7 \%)$ & $16(55.2 \%)$ \\
LEC1 & $10(38.5 \%)$ & $11(47.8 \%)$ & $5(41.7 \%)$ & $12(41.4 \%)$ \\
LEC2 & $1(3.8 \%)$ & $1(4.3 \%)$ & $1(8.3 \%)$ & $2(6.9 \%)$ \\
PLT1 & $8(30.8 \%)$ & $8(34.85)$ & $3(25.0 \%)$ & $11(37.9 \%)$ \\
PLT2 & $8(30.8 \%)$ & $14(60.9 \%)$ & $6(50.0 \%)$ & $14(48.3 \%)$ \\
PLT3 & $5(19.2 \%)$ & $10(43.5 \%)$ & $1(8.3 \%)$ & $12(41.4 \%)$ \\
PLT7 & $10(38.5 \%)$ & $7(30.4 \%)$ & $3(25.0 \%)$ & $9(31.0 \%)$ \\
\hline
\end{tabular}


Table 4. Cont.

\begin{tabular}{ccccc}
\hline \multirow{2}{*}{ SE Related Genes } & \multicolumn{4}{c}{ Number (Percentage) of Co-Expressed Auxin Early Response Genes } \\
\cline { 2 - 5 } & ARF & AuxIAA & GH3 & SAUR \\
\hline BBM & $2(7.7 \%)$ & $1(4.3 \%)$ & $1(8.3 \%)$ & $2(6.9 \%)$ \\
PLT5 & $12(46.2 \%)$ & $17(73.9 \%)$ & $7(58.3 \%)$ & $16(55.2 \%)$ \\
WUS & $0(0.0 \%)$ & $0(0.0 \%)$ & $0(0.0 \%)$ & $0(0.0 \%)$ \\
SERK3 & $9(34.6 \%)$ & $5(21.7 \%)$ & $3(25.0 \%)$ & $9(31.0 \%)$ \\
SERK4 & $8(30.8 \%)$ & $10(43.5 \%)$ & $7(58.3 \%)$ & $15(51.7)$ \\
Total & 26 & 23 & 12 & 29 \\
\hline
\end{tabular}

We constructed four networks to show the co-expression relations between somatic embryogenesis related genes and the auxin early response gene families $A R F, A u x / I A A, G H 3$, and $S A U R$, respectively (Figure 7). Results showed that each auxin early response gene may co-express with multiple different somatic embryogenesis related genes, and vice versa, each somatic embryogenesis related gene may co-express with different auxin early response genes. The majority of co-expressed relations between somatic embryogenesis related genes and the $A R F$ and $G H 3$ family genes were positive, while the $A u x / I A A$ and $S A U R$ families had a greater negative relation with somatic embryogenesis related genes (Figure 7).
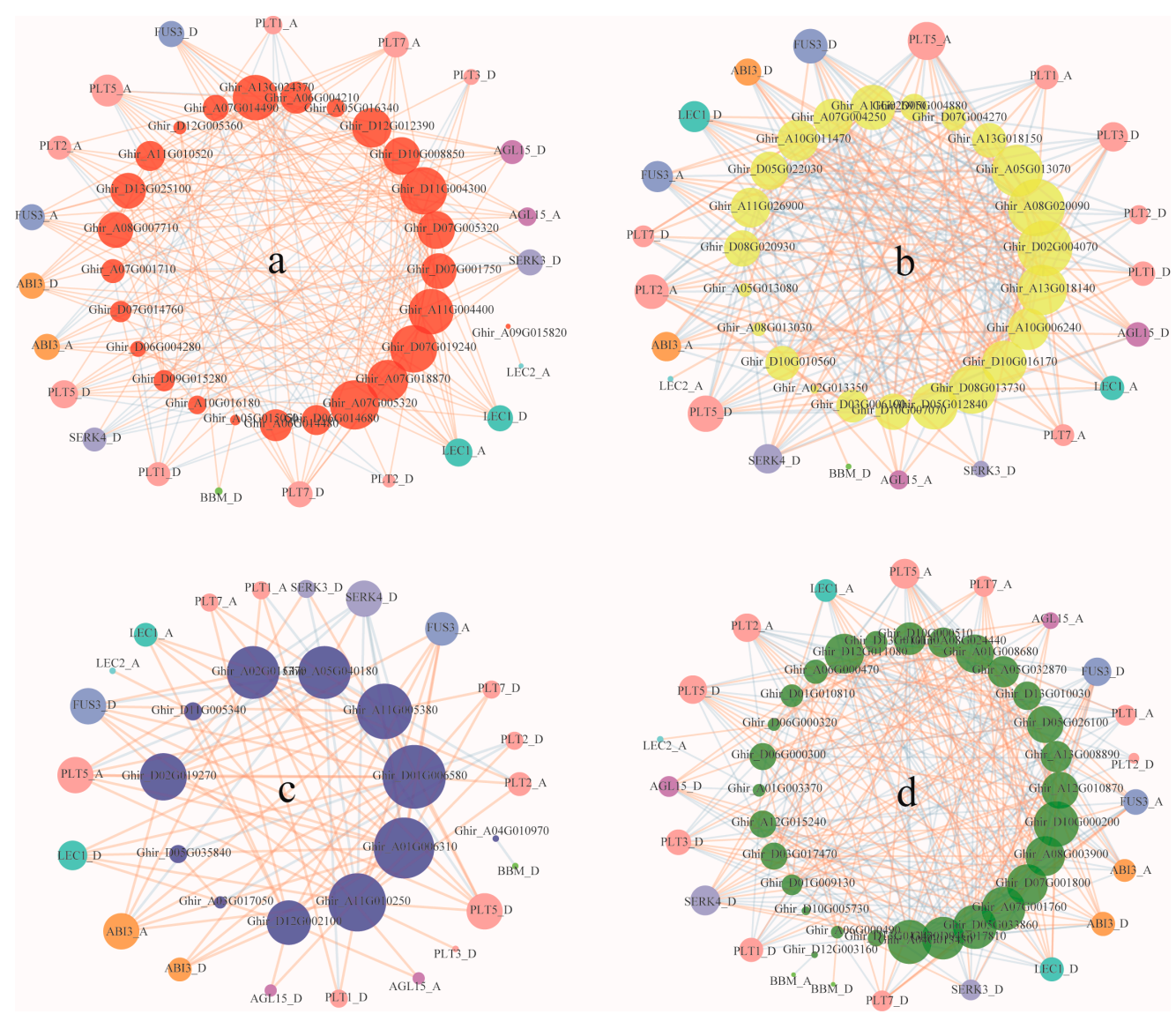

Figure 7. Co-expression networks of somatic embryogenesis related genes and four auxin early response gene families $A R F(\mathbf{a}), A u x / I A A(\mathbf{b}), G H 3(\mathbf{c})$, and SAUR (d). Reported somatic embryogenesis related genes ABI3, AGL15, FUS3, LEC1, LEC2, PLTS, BBM, and SERKs were identified in G. hirsutum and are represented by circle nodes in different colors. The red line links two nodes, indicating a positive correlation between these two genes, while the blue line indicates a negative correlation between genes. Only the intercorrelationship between auxin early response genes and somatic embryogenesis related genes and the intracorrelationship in auxin early response genes are shown. The size of nodes represents the degree of co-expression relations. 
In the ARF family, genes co-expressed with most somatic embryogenesis related genes were positively correlated with some exceptions. SERK4_D and PLT1_A were principally co-expressed with the $A R F$ genes negatively. Half of the PLT1_D and PLT2_D were co-expressed and a few ABI3_A, FUS3_D, PLT2_A, PLT5, PLT3_D, and SERK3_D co-expressed ARF genes were negatively correlated with these somatic embryogenesis related genes (Figure 7a). Similar results were found in the GH3 family, as most somatic embryogenesis related genes were positively correlated with GH3 genes, but SERK4_D was negatively correlated with GH3 genes (except for Ghir_A05G040180). Furthermore, ABI3_A, FUS3_D, PLT1_A,PLT1_D, PLT2_A, PLT2_D, PLT5_A, PLT5_D, and SERK3_D sometimes showed a negative correlation with some of the co-expressed GH3 genes (Figure 7c).

In the $A u x / I A A$ and SAUR families, genes co-expressed with somatic embryogenesis related genes in complex ways: somatic embryogenesis related genes positively and negatively correlated with $A u x / I A A$ or SAUR family genes at the same times (Figure $7 \mathrm{~b}, \mathrm{~d}$ ). Nevertheless, we found that PLT2_D was principally negatively correlated with $A u x / I A A$ genes and absolutely negatively correlated with SAUR genes, while PLT1_A was principally negatively correlated with SAUR genes.

\section{Discussion}

Auxin early response genes that play a key component in auxin homeostasis maintenance and signaling have been widely characterized in many plants. There were 23 ARFs, 29 Aux/IAAs, 20 GH3s, and 79 SAURs identified in Arabidopsis [17,22,61-63]; 17 ARFs, 26 Aux/IAAs, 15 GH3s, and 99 SAURs in tomato [64-67]; 22 ARFs, 26 Aux/IAAs, 11 GH3s, and 70 SAURs in citrus [68]; 16 ARFs, 27 Aux/IAAs, 10 GH3s, and 61 SAURs in cucumber [69]; 25 ARFs, 31 Aux/IAAs, 12 GH3s, and 58 SAURs in rice [70-73]; and 35 ARFs, 31 Aux/IAAs, 13 GH3s, and 79 SAURs in maize [74-77]. In the present study, 71 ARFs, 86 Aux/IAAs, 63 GH3s, and 194 SAURs were identified in upland cotton. All four auxin early response gene families had more gene members in upland cotton than many other plants, which may be largely attributed to the genome duplication of the allotetraploid upland cotton, thought to be derived from the allopolyploidization of A-subgenome and D-subgenome, in line with the result that segmental duplications widely exist in the upland cotton $A R F$ family. Half the number of upland cotton $A R F$ genes was nearly equal to that of maize $(\sim 2.3 \mathrm{G})$, which has a similar size of genome with upland cotton $(\sim 2.5 \mathrm{G})$, but about 1.5 fold of that in Arabidopsis, suggesting that the ARF family in upland cotton was relatively conserved when compared to maize, but somewhat expanded when compared to Arabidopsis. The number of upland cotton $A u x / I A A$ genes was about 2.8- and 3-folds of that in maize and Arabidopsis, respectively, implying the obvious expansion of the $A u x / I A A$ family in upland cotton. Only two genes were found to be involved in tandem duplication, while segmental duplication was extensively occurred. Therefore, genome duplication and segmental duplication played important roles in the expansion of $A u x / I A A$ in upland cotton. The GH3 and SAUR families were largely expanded in upland cotton when compared to many other plants. For the GH3 family, there were $13 \mathrm{GH} 3$ genes derived from tandem duplication in upland cotton, which may contribute to the expansion of the GH3 family in some degree. Nevertheless, when these tandem duplicates were excluded, the number of remaining GH3 gene members in upland cotton was still higher than twice of that in Arabidopsis. Collinearity analysis revealed that segmental duplication also extensively contributed to GH3 family expansion. Further collinearity investigation found that the two GH3 gene members, Ghir_A03G020320 and Ghir_D02G021740, were subjected to extensive segmental duplication as both genes had multiple corresponding syntenic genes on different chromosomes (chromosome 5 and 4, respectively). Phylogeny revealed that these two genes were orthologous genes of AtGH3.1 (AT2G14960), which encoded a protein similar to IAA-amino synthases [22]. However, transcription profiles showed that only half of the two AtGH3.1-like genes and their homologs were actively expressed during the embryogenic potency acquisition of cotton callus. This implies the functional divergence of these gene duplicates during evolution. Aside from genome duplication, the SAUR family expansion can be largely attributed by the tandem duplication in upland cotton, where each gene is more likely to repeat many times (Figure 2a). 
As tandem duplication extensively existed in the two greatly expanded GH3 and SAUR families, the involved genes were further investigated. The majority of tandem duplicates derived from $G H 3$ genes were distributed in Group II, which was likely to be upland cotton specific that contained no Arabidopsis gene (Figure 1c), implying that the expansion of the GH3 family in upland cotton may result from the divergent evolution of Arabidopsis and upland cotton, largely bringing new specific functions for upland cotton. Other $G H 3$ tandem duplicates involving eight genes belonged to Groups IV and V. The four GH3 tandem duplicates in Group IV were orthologous genes of AtGH3.11 (AT2G46370), also named AtJAR1, which acts as a positive regulator of JA signaling by encoding a jasmonate-amino synthetase that catalyzes jasmonyl-isoleucine (JA-Ile) conjugation, then promotes the interaction between JAZ1 repressor and COI1 $[78,79]$. A functional study revealed that AtJAR1 was involved in pathogen defense and wound responses. Thus, the duplication of AtJAR1 homologous genes in upland cotton may enhance its JA signaling and confer resistance to diverse biotic and abiotic stresses. The four GH3 tandem duplicates in Group V were orthologous genes of AtGH3.5 and $A t G H 3.6$, encoded IAA-amino synthases, and participated in auxin homeostasis maintenance. Almost all tandem duplicated SAUR genes in upland cotton were distributed in Groups III, V, and VII. Tandem duplicates in Group III were orthologous genes of AtSAUR1-AtSAUR5 with unknown function. Transcription profiles showed that only some of them were actively expressed during the embryogenic potency acquisition of cotton callus, implying that these duplicates may function in different developmental and physiological processes. Tandem duplicates in Group V and VII were orthologous genes of AtSAUR61-AtSAUR68 and AtSAUR48, respectively; however, all these tandem duplicated genes showed a low expression level during the embryogenic potency acquisition of cotton callus, so we speculated that they may function in other physiological processes.

To investigate the roles of auxin early response genes in upland cotton somatic embryogenesis, we profiled their expression patterns during embryogenic potency acquisition of the callus. The ARF and $A u x / I A A$ family genes were extensively expressed in this process, while only a small part of the GH3 and SAUR family genes was active. Furthermore, more than $60 \%$ of the ARF and Aux/IAA family genes showed differential expression during the process from NEC to EC, suggesting that the $A R F$ and $A u x / I A A$ family play crucial roles in the regulation of auxin-mediated somatic embryogenesis in upland cotton. Though only the minority of GH3 and SAUR genes showed active expression in embryogenic potency acquisition, the majority of these active genes were differentially expressed in NEC compared to EC, implying their regulatory roles in upland cotton somatic embryogenesis. When compared, the expression profiles of the auxin early response genes between cultivars with different regeneration abilities, the $A R F$ and GH3 genes were more likely to exhibit comparable expression patterns, while about half of the $A u x / I A A$ and SAUR genes did not. Typically, during the transformation from NEC to EC, the Aux/IAA gene Ghir_A05G002590 and the SAUR gene Ghir_A03G022510 were almost specifically expressed in the NEC of CCRI24, and downregulated significantly into low expression levels in CCRI24, while maintaining low expression levels throughout the transformation in CCRI12 (Figure 5). The high accumulation of transcripts of these two genes in the NEC may be conducive to initiating the embryogenic transformation into EC. Subsequent significant decreased expression of these genes may be tightly regulated by the sharply increasing IAA content in the callus. Additionally, it is noteworthy that the Aux/IAA gene Ghir_D05G002730 exhibited obvious opposite expressed trends between CCRI12 and CCRI24 during callus embryogenic potency acquisition as significantly upregulated in CCRI12, but downregulated in CCRI24 (Figure 5). The different expression patterns of this gene between CCRI12 and CCRI24 may be associated with the difference of their somatic embryogenesis abilities. Further expression profiles analysis found that this gene had a significantly higher expression level in the NEC of CCRI24 than that of CCRI12, just like its homologous gene Ghir_A05G002590 as described above. Therefore, we speculated that these genes may be negatively correlated with the transformation from NEC to EC, but be of benefit for triggering embryogenic transformation at high expression level. Overexpression of these genes in NEC may help improve the efficiency of somatic embryogenesis in reluctant cotton lines. Based on the results of enrichment analysis, the ARF family was enriched 
in upregulated genes during callus embryogenic potency acquisition in both CCRI12 and CCRI24 (Figure 6), suggesting the general positive regulatory roles of the ARF family in the embryogenic potency acquisition of upland cotton callus. The $A u x / I A A$ family was generally downregulated during callus embryogenic potency acquisition in CCRI24, while enriched in downregulated genes in CCRI12. At the same time, the $A u x / I A A$ family was generally significantly upregulated in the NEC of CCRI24 when compared to that of CCRI12 (Figure 6). This suggests that the overall high expression level of the Aux/IAA family genes or some critical gene members in NEC may be a prerequisite for the initiation of the embryogenic transformation into $\mathrm{EC}$, even though there was subsequent downregulation during the transformation, as above-mentioned. Furthermore, the SAUR family, as effectors of hormone signaling [25], was enriched in CCRI24, but not in CCRI12 during transformation from NEC to EC, indicating the more effective regulation of auxin signaling in CCRI24.

Auxin early response genes in the $A R F, A u x / I A A$, and $S A U R$ family were tightly co-expressed during the embryogenic potency acquisition of upland cotton callus, suggesting the close coordination of these genes in auxin signaling mediated somatic embryogenic transformation. About half of the differentially expressed $A R F, A u x / I A A, G H 3$, and SAUR genes were co-expressed with previously reported somatic embryogenesis related genes, implying the associated regulation between them. The ARF and GH3 family genes are likely to show a synergistic effect with somatic embryogenesis related genes as there is a generally positive correlation between them. Auxin early response genes were found co-expressed with most of the known somatic embryogenesis related genes including the ABI3, FUS3, LEC1, PLTS (PLT1, PLT2, PLT3, PLT5, PLT7), AGL15, and SERKs (SERK3 and SERK4) genes. We speculate that these somatic embryogenesis related genes are implicated in upland cotton somatic embryogenesis by regulating or responding to auxin early response genes, or are mediated by ARF, $A u x / I A A, G H 3$, and SAUR gene-involved auxin signaling up to some extent. While no auxin early response gene was co-expressed with WUS, it was supposed that WUS may regulate upland cotton somatic embryogenesis through other pathways, or directly regulated target genes downstream of the auxin signaling. In the present study, auxin early response genes were revealed to be co-expressed with a number of PLT genes during the embryogenic potency acquisition of upland cotton callus. Different $P L T$ gene members showed a different co-expression pattern with auxin early response, where there were obviously more auxin early response genes co-expressed with PLT2 and PLT5 than PLT1 and $P L T 7$, while only a few auxin early response genes were co-expressed with PLT4/BBM. Furthermore, PLT3 is more likely to be co-expressed with $A u x / I A A$ and SAUR genes than ARF and Aux/IAA genes. These results suggest the complex regulatory patterns of PLT genes in somatic embryogenesis. Even though there is functional overlap and redundancy of PLT genes in plant development, each PLT gene member had specific co-regulation or response patterns during somatic embryogenesis. Previous studies have identified some PLT genes (PLT1 and PLT2) as late auxin response genes and necessary for zygotic embryo development $[80,81]$. PLT2 and PLT4/BBM induced somatic embryogenesis mediated by auxin signaling [56,82], while the functional and molecular mechanism of PLT genes in somatic embryogenesis remain unclear. The co-expression findings in our study provide valuable information for the exploration of the functional mechanism of PLTs in somatic embryogenesis.

\section{Conclusions}

In the present study, we conducted a genome-wide identification and comprehensive phylogenetic analysis of auxin early response genes in upland cotton including $A R F, A u x / I A A, G H 3$, and SAUR family members, which act as a key component of auxin signaling. The transcriptional dynamics of these auxin early response genes during embryogenic competence acquisition of cotton callus were further focused, based on transcriptomic analysis. The results revealed that auxin early response genes were extensively involved and played important roles in the embryogenic transformation of cotton callus. The expression level difference of the $A u x / I A A$ family genes between different cultivars may be associated with their difference in somatic embryogenesis capacity. Co-expression analysis implied 
that most previously reported somatic embryogenesis related genes were implicated in plant somatic embryogenesis by interacting with auxin early response genes.

Supplementary Materials: The following are available online at http://www.mdpi.com/2073-4425/10/10/730/s1, Figure S1. Multiple sequence alignment of ARF proteins from Arabidopsis and upland cotton showed conserved residues or motifs. Figure S2. Multiple sequence alignment of Aux/IAA proteins from Arabidopsis and upland cotton showed conserved residues or motifs. Figure S3. Multiple sequence alignment of GH3 proteins from Arabidopsis and upland cotton showed conserved residues or motifs. Figure S4. Multiple sequence alignment of SAUR proteins from Arabidopsis and upland cotton showed conserved residues or motifs. Figure S5. Domain and exon-intron organization structure of identified $A R F(\mathbf{a}), A u x / I A A(\mathbf{b}), G H 3(\mathbf{c})$, SAUR (d) family gene members in Arabidopsis and upland cotton. Figure S6. Co-expression network showing the tight correlationship among the four auxin early response families. Table S1. The summary of expression of auxin early response genes during the transformation from NEC to EC. Table S2. Primers used for qRT-PCR validation of expression pattern of auxin early response genes.

Author Contributions: Conceptualization, R.S.; Data curation, Y.L.; Formal analysis, R.S.; Investigation, R.S.; Methodology, R.S.; Project administration, C.L.; Resources, D.M.; Supervision, C.L.; Visualization, S.W.; Writing-original draft, R.S.

Funding: This work was supported by the National Science and Technology Major Project of China (2016YFD0101006).

Conflicts of Interest: The authors declare no conflicts of interest.

\section{References}

1. Shi, Y.-H.; Zhu, S.-W.; Mao, X.-Z.; Feng, J.-X.; Qin, Y.-M.; Zhang, L.; Cheng, J.; Wei, L.-P.; Wang, Z.-Y.; Zhu, Y.-X. Transcriptome profiling, molecular biological, and physiological studies reveal a major role for ethylene in cotton fiber cell elongation. Plant Cell 2006, 18, 651-664. [CrossRef] [PubMed]

2. Sunilkumar, G.; Campbell, L.M.; Puckhaber, L.; Stipanovic, R.D.; Rathore, K.S. Engineering cottonseed for use in human nutrition by tissue-specific reduction of toxic gossypol. PNAS 2006, 103, 18054-18059. [CrossRef] [PubMed]

3. Guo, S.; Wang, Y.; Sun, G.; Jin, S.; Zhou, T.; Meng, Z.; Zhang, R. Twenty years of research and application of transgenic cotton in China. Scientia Agricultura Sinica 2015, 48, 3372-3387.

4. Liu, C.; Tian, R.; Kong, D.; Li, F.; Shang, H.; Chen, X. Establishment and application of efficient transformation system for cotton. Scientia Agricultura Sinica 2014, 47, 4183-4197.

5. Zhang, B.-H.; Liu, F.; Yao, C.-B. Plant regeneration via somatic embryogenesis in cotton. Plant Cell Tissue Organ Cult. 2000, 60, 89-94. [CrossRef]

6. Leelavathi, S.; Sunnichan, V.G.; Kumria, R.; Vijaykanth, G.P.; Bhatnagar, R.K.; Reddy, V.S. A simple and rapid Agrobacterium -mediated transformation protocol for cotton (Gossypium hirsutum L.): Embryogenic calli as a source to generate large numbers of transgenic plants. Plant Cell Rep. 2004, 22, 465-470. [CrossRef]

7. Li, J.; Wang, M.; Li, Y.; Zhang, Q.; Lindsey, K.; Daniell, H.; Jin, S.; Zhang, X. Multi-omics analyses reveal epigenomics basis for cotton somatic embryogenesis through successive regeneration acclimation (SRA) process. Plant Biotechnol. J. 2019, 17, 435-450. [CrossRef]

8. Rajeswari, S.; Muthuramu, S.; Chandirakala, R.; Thiruvengadam, V.; Raveendran, T.S. Callus induction, somatic embryogenesis and plant regeneration in cotton (Gossypium hirsutum L.). Electron. J. Plant Breed. 2010, 1, 1186-1190.

9. Jin, S.; Zhang, X.; Liang, S.; Nie, Y.; Guo, X.; Huang, C. Factors affecting transformation efficiency of embryogenic callus of Upland cotton (Gossypium hirsutum) with Agrobacterium tumefaciens. Plant Cell Tissue Organ Cult. 2005, 81, 229-237. [CrossRef]

10. Zhang, C.J.; Yu, S.X.; Fan, S.L.; Zhang, J.F.; Li, F.G. Inheritance of somatic embryogenesis using leaf petioles as explants in upland cotton. Euphytica 2011, 181, 55-63. [CrossRef]

11. Deo, P.C.; Tyagi, A.P.; Taylor, M.; Harding, R.; Becker, D. Factors affecting somatic embryogenesis and transformation in modern plant breeding. S. Pac. J. Nat. App. Sci. 2010, 28, 27. [CrossRef]

12. Xu, Z.; Zhang, C.; Zhang, X.; Liu, C.; Wu, Z.; Yang, Z.; Zhou, K.; Yang, X.; Li, F. Transcriptome profiling reveals auxin and cytokinin regulating somatic embryogenesis in different sister lines of cotton cultivar CCRI24. J. Integr. Plant Biol. 2013, 55, 631-642. [CrossRef] [PubMed] 
13. Yang, X.; Zhang, X.; Yuan, D.; Jin, F.; Zhang, Y.; Xu, J. Transcript profiling reveals complex auxin signalling pathway and transcription regulation involved in dedifferentiation and redifferentiation during somatic embryogenesis in cotton. BMC Plant Biol. 2012, 12, 110. [CrossRef] [PubMed]

14. Zeng, F.; Zhang, X.; Jin, S.; Cheng, L.; Liang, S.; Hu, L.; Guo, X.; Nie, Y.; Cao, J. Chromatin reorganization and endogenous auxin/cytokinin dynamic activity during somatic embryogenesis of cultured cotton cell. Plant Cell Tissue Organ Cult. 2007, 90, 63-70. [CrossRef]

15. Abel, S.; Theologis, A. Early genes and auxin action. Plant Physiol. 1996, 111, 9-17. [CrossRef]

16. Chapman, E.J.; Estelle, M. Mechanism of auxin-regulated gene expression in plants. Annu. Rev. Genetics 2009, 43, 265-285. [CrossRef] [PubMed]

17. Hagen, G.; Guilfoyle, T. Auxin-responsive gene expression: Genes, promoters and regulatory factors. Plant Mol. Biol. 2002, 49, 373-385. [CrossRef]

18. Tiwari, S.B. The roles of auxin response factor domains in auxin-responsive transcription. Plant Cell 2003, 15, 533-543. [CrossRef]

19. Luo, J.; Zhou, J.-J.; Zhang, J.-Z. Aux/IAA gene family in plants: Molecular structure, regulation, and function. Int. J. Mole. Sci. 2018, 19, 259. [CrossRef]

20. Tiwari, S.B.; Wang, X.-J.; Hagen, G.; Guilfoyle, T.J. AUX/IAA proteins are active repressors, and their stability and activity are modulated by auxin. Plant Cell 2001, 13, 2809-2822. [CrossRef]

21. Gray, W.M.; Kepinski, S.; Rouse, D.; Leyser, O.; Estelle, M. Auxin regulates SCFTIR1-dependent degradation of AUX/IAA proteins. Nature 2001, 414, 271-276. [CrossRef] [PubMed]

22. Staswick, P.E. Characterization of an Arabidopsis enzyme family that conjugates amino acids to indole-3-acetic acid. Plant Cell 2005, 17, 616-627. [CrossRef] [PubMed]

23. Franco, A.R.; Gee, M.A.; Guilfoyle, T.J. Induction and superinduction of auxin-responsive mRNAs with auxin and protein synthesis inhibitors. J. Biol. Chem. 1990, 265, 15845-15849. [PubMed]

24. McClure, B.; Guilfoyle, T. Rapid redistribution of auxin-regulated RNAs during gravitropism. Science 1989, 243, 91-93. [CrossRef] [PubMed]

25. Ren, H.; Gray, W.M. SAUR proteins as effectors of hormonal and environmental signals in plant growth. Mol. Plant 2015, 8, 1153-1164. [CrossRef] [PubMed]

26. Cao, A.; Zheng, Y.; Yu, Y.; Wang, X.; Shao, D.; Sun, J.; Cui, B. Comparative transcriptome analysis of SE initial dedifferentiation in cotton of different SE capability. Sci. Rep. 2017, 7, 8583. [CrossRef]

27. Jin, F.; Hu, L.; Yuan, D.; Xu, J.; Gao, W.; He, L.; Yang, X.; Zhang, X. Comparative transcriptome analysis between somatic embryos (SEs) and zygotic embryos in cotton: Evidence for stress response functions in SE development. Plant Biotechnol. J. 2014, 12, 161-173. [CrossRef]

28. Sun, R.; Tian, R.; Ma, D.; Wang, S.; Liu, C. Comparative transcriptome study provides insights into acquisition of embryogenic ability in upland cotton during somatic embryogenesis. J. Cotton Res. 2018, 1, 9. [CrossRef]

29. Wang, M.; Tu, L.; Yuan, D.; Zhu, D.; Shen, C.; Li, J.; Liu, F.; Pei, L.; Wang, P.; Zhao, G.; et al. Reference genome sequences of two cultivated allotetraploid cottons, Gossypium hirsutum and Gossypium barbadense. Nat. Genet. 2019, 51, 224-229. [CrossRef]

30. El-Gebali, S.; Mistry, J.; Bateman, A.; Eddy, S.R.; Luciani, A.; Potter, S.C.; Qureshi, M.; Richardson, L.J.; Salazar, G.A.; Smart, A.; et al. The Pfam protein families database in 2019. Nucleic Acids Res. 2019, 47, D427-D432. [CrossRef]

31. Finn, R.D.; Clements, J.; Eddy, S.R. HMMER web server: Interactive sequence similarity searching. Nucleic Acids Res. 2011, 39, W29-W37. [CrossRef] [PubMed]

32. Camacho, C.; Coulouris, G.; Avagyan, V.; Ma, N.; Papadopoulos, J.; Bealer, K.; Madden, T.L. BLAST+: Architecture and applications. BMC Bioinforma. 2009, 10, 421. [CrossRef] [PubMed]

33. Jones, P.; Binns, D.; Chang, H.-Y.; Fraser, M.; Li, W.; McAnulla, C.; McWilliam, H.; Maslen, J.; Mitchell, A.; Nuka, G.; et al. InterProScan 5: Genome-scale protein function classification. Bioinformatics 2014, 30, 1236-1240. [CrossRef] [PubMed]

34. Edgar, R.C. MUSCLE: Multiple sequence alignment with high accuracy and high throughput. Nucleic Acids Res. 2004, 32, 1792-1797. [CrossRef] [PubMed]

35. Trifinopoulos, J.; Nguyen, L.-T.; von Haeseler, A.; Minh, B.Q. W-IQ-TREE: A fast online phylogenetic tool for maximum likelihood analysis. Nucleic Acids Res. 2016, 44, W232-W235. [CrossRef] [PubMed]

36. Hao, Z.; Lv, D.; Ge, Y.; Shi, J.; Yu, G.; Chen, J. RIdeogram: Drawing SVG graphics to visualize and map genome-wide data in idiograms. PeerJ Preprints 2019. [CrossRef] 
37. Wang, Y.; Tang, H.; Debarry, J.D.; Tan, X.; Li, J.; Wang, X.; Lee, T.H.; Jin, H.; Marler, B.; Guo, H.; et al. MCScanX: A toolkit for detection and evolutionary analysis of gene synteny and collinearity. Nucleic Acids Res. 2012, 40, e49. [CrossRef]

38. Krzywinski, M.; Schein, J.; Birol, I.; Connors, J.; Gascoyne, R.; Horsman, D.; Jones, S.J.; Marra, M.A. Circos: An information aesthetic for comparative genomics. Genome Res. 2009, 19, 1639-1645. [CrossRef]

39. Kim, D.; Langmead, B.; Salzberg, S.L. HISAT: A fast spliced aligner with low memory requirements. Nat. Methods 2015, 12, 357-360. [CrossRef]

40. Pertea, M.; Pertea, G.M.; Antonescu, C.M.; Chang, T.-C.; Mendell, J.T.; Salzberg, S.L. StringTie enables improved reconstruction of a transcriptome from RNA-seq reads. Nat. Biotechnol. 2015, 33, 290-295. [CrossRef]

41. Love, M.I.; Huber, W.; Anders, S. Moderated estimation of fold change and dispersion for RNA-seq data with DESeq2. Genome Biol. 2014, 15, 550. [CrossRef] [PubMed]

42. Yu, G.; Wang, L.-G.; Han, Y.; He, Q.-Y. clusterProfiler: An R package for comparing biological themes among gene clusters. OMICS 2012, 16, 284-287. [CrossRef] [PubMed]

43. Croken, M.; Qiu, W.; White, M.W.; Kim, K. Gene Set Enrichment Analysis (GSEA) of Toxoplasma gondii expression datasets links cell cycle progression and the bradyzoite developmental program. BMC Genomics 2014, 15, 515. [CrossRef] [PubMed]

44. Shannon, P. Cytoscape: A software environment for integrated models of biomolecular interaction networks. Genome Res. 2003, 13, 2498-2504. [CrossRef] [PubMed]

45. Guilfoyle, T.J.; Hagen, G. Auxin response factors. Cur. Opin. Plant Biol. 2007, 10, 453-460. [CrossRef] [PubMed]

46. Ramos, J.A.; Zenser, N.; Leyser, O.; Callis, J. Rapid degradation of auxin/indoleacetic acid proteins requires conserved amino acids of domain II and is proteasome dependent. Plant Cell 2001, 13, 2349. [CrossRef] [PubMed]

47. Moss, B.L.; Mao, H.; Guseman, J.M.; Hinds, T.R.; Hellmuth, A.; Kovenock, M.; Noorassa, A.; Lanctot, A.; Villalobos, L.I.A.C.; Zheng, N.; et al. Rate motifs tune auxin/indole-3-acetic acid degradation dynamics. Plant Physiol. 2015, 169, 803-813. [CrossRef]

48. Yu, D.; Qanmber, G.; Lu, L.; Wang, L.; Li, J.; Yang, Z.; Liu, Z.; Li, Y.; Chen, Q.; Mendu, V.; et al. Genome-wide analysis of cotton GH3 subfamily II reveals functional divergence in fiber development, hormone response and plant architecture. BMC Plant Biol. 2018, 18. [CrossRef]

49. Sun, R.; Wang, K.; Guo, T.; Jones, D.C.; Cobb, J.; Zhang, B.; Wang, Q. Genome-wide identification of auxin response factor $(\mathrm{ARF})$ genes and its tissue-specific prominent expression in Gossypium raimondii. Funct. Integr. Genomics 2015, 15, 481-493. [CrossRef]

50. Xiao, G.; He, P.; Zhao, P.; Liu, H.; Zhang, L.; Pang, C.; Yu, J. Genome-wide identification of the GhARF gene family reveals that GhARF2 and GhARF18 are involved in cotton fibre cell initiation. J. Exp. Bot. 2018, 69, 4323-4337. [CrossRef]

51. Zhang, T.; Hu, Y.; Jiang, W.; Fang, L.; Guan, X.; Chen, J.; Zhang, J.; Saski, C.A.; Scheffler, B.E.; Stelly, D.M.; et al. Sequencing of allotetraploid cotton (Gossypium hirsutum L. acc. TM-1) provides a resource for fiber improvement. Nature Biotechnol. 2015, 33, 531. [CrossRef] [PubMed]

52. Li, X.; Liu, G.; Geng, Y.; Wu, M.; Pei, W.; Zhai, H.; Zang, X.; Li, X.; Zhang, J.; Yu, S.; et al. A genome-wide analysis of the small auxin-up RNA (SAUR) gene family in cotton. BMC Genomics 2017, 18. [CrossRef] [PubMed]

53. Ahmadi, B.; Masoomi-Aladizgeh, F.; Shariatpanahi, M.E.; Azadi, P.; Keshavarz-Alizadeh, M. Molecular characterization and expression analysis of SERK1 and SERK2 in Brassica napus L.: Implication for microspore embryogenesis and plant regeneration. Plant Cell Rep. 2016, 35, 185-193. [CrossRef] [PubMed]

54. Elhiti, M.; Stasolla, C.; Wang, A. Molecular regulation of plant somatic embryogenesis. In Vitro Cell. Dev. Biol., Plant 2013, 49, 631-642. [CrossRef]

55. Horstman, A.; Bemer, M.; Boutilier, K. A transcriptional view on somatic embryogenesis. Regeneration 2017, 4, 201-216. [CrossRef] [PubMed]

56. Horstman, A.; Li, M.; Heidmann, I.; Weemen, M.; Chen, B.; Muiño, J.M.; Angenent, G.C.; Boutilier, K. The BABY BOOM transcription factor activates the LEC1-ABI3-FUS3-LEC2 network to induce somatic embryogenesis. Plant Physiol. 2017, 175, 848-857. [CrossRef] 
57. Bouchabke-Coussa, O.; Obellianne, M.; Linderme, D.; Montes, E.; Maia-Grondard, A.; Vilaine, F.; Pannetier, C. Wuschel overexpression promotes somatic embryogenesis and induces organogenesis in cotton (Gossypium hirsutum L.) tissues cultured in vitro. Plant Cell Rep. 2013, 32, 675-686. [CrossRef]

58. Su, Y.H.; Zhao, X.Y.; Liu, Y.B.; Zhang, C.L.; O'Neill, S.D.; Zhang, X.S. Auxin-induced WUS expression is essential for embryonic stem cell renewal during somatic embryogenesis in Arabidopsis. Plant J. 2009, 59, 448-460. [CrossRef]

59. Zhang, S.; Liu, X.; Lin, Y.; Xie, G.; Fu, F.; Liu, H.; Wang, J.; Gao, S.; Lan, H.; Rong, T. Characterization of a ZmSERK gene and its relationship to somatic embryogenesis in a maize culture. Plant Cell Tissue Organ Cult. 2011, 105, 29-37. [CrossRef]

60. Zheng, W.; Zhang, X.; Yang, Z.; Wu, J.; Li, F.; Duan, L.; Liu, C.; Lu, L.; Zhang, C.; Li, F. AtWuschel promotes formation of the embryogenic callus in Gossypium hirsutum. PLoS ONE 2014, 9, e87502. [CrossRef]

61. Gil, P.; Liu, Y.; Orbovic, V.; Verkamp, E.; Poff, K.L.; Green, P.J. Characterization of the auxin-inducible SAUR-AC1 gene for use as a molecular genetic tool in Arabidopsis. Plant Physiol. 1994, 104, 777-784. [CrossRef] [PubMed]

62. Okushima, Y. Functional genomic analysis of the AUXIN RESPONSE FACTOR gene family members in Arabidopsis thaliana: Unique and overlapping functions of ARF7 and ARF19. Plant Cell 2005, 17, 444-463. [CrossRef] [PubMed]

63. Remington, D.L. Contrasting modes of diversification in the Aux/IAA and ARF gene families. Plant Physiol. 2004, 135, 1738-1752. [CrossRef] [PubMed]

64. Kumar, R.; Tyagi, A.K.; Sharma, A.K. Genome-wide analysis of auxin response factor (ARF) gene family from tomato and analysis of their role in flower and fruit development. Mol. Genetics Genomics 2011, 285, 245-260. [CrossRef] [PubMed]

65. Kumar, R.; Agarwal, P.; Tyagi, A.K.; Sharma, A.K. Genome-wide investigation and expression analysis suggest diverse roles of auxin-responsive GH3 genes during development and response to different stimuli in tomato (Solanum lycopersicum). Mol. Genetics Genomics 2012, 287, 221-235. [CrossRef] [PubMed]

66. Wu, J.; Liu, S.; He, Y.; Guan, X.; Zhu, X.; Cheng, L.; Wang, J.; Lu, G. Genome-wide analysis of SAUR gene family in Solanaceae species. Gene 2012, 509, 38-50. [CrossRef] [PubMed]

67. Wu, J.; Peng, Z.; Liu, S.; He, Y.; Cheng, L.; Kong, F.; Wang, J.; Lu, G. Genome-wide analysis of Aux/IAA gene family in Solanaceae species using tomato as a model. Mol. Genet Genomics 2012, 287, 295-311. [CrossRef] [PubMed]

68. Xie, R.; Dong, C.; Ma, Y.; Deng, L.; He, S.; Yi, S.; Lv, Q.; Zheng, Y. Comprehensive analysis of SAUR gene family in citrus and its transcriptional correlation with fruitlet drop from abscission zone A. Funct. Integr. Genomics 2015, 15, 729-740. [CrossRef]

69. Wu, J.; Liu, S.; Guan, X.; Chen, L.; He, Y.; Wang, J.; Lu, G. Genome-wide identification and transcriptional profiling analysis of auxin response-related gene families in cucumber. BMC Res. Notes 2014, 7, 218. [CrossRef]

70. Jain, M.; Kaur, N.; Garg, R.; Thakur, J.K.; Tyagi, A.K.; Khurana, J.P. Structure and expression analysis of early auxin-responsive Aux/IAA gene family in rice (Oryza sativa). Funct. Integr. Genomics 2006, 6, 47-59. [CrossRef]

71. Jain, M.; Kaur, N.; Tyagi, A.K.; Khurana, J.P. The auxin-responsive GH3 gene family in rice (Oryza sativa). Funct. Integr. Genomics 2006, 6, 36-46. [CrossRef] [PubMed]

72. Jain, M.; Tyagi, A.K.; Khurana, J.P. Genome-wide analysis, evolutionary expansion, and expression of early auxin-responsive SAUR gene family in rice (Oryza sativa). Genomics 2006, 88, 360-371. [CrossRef] [PubMed]

73. Wang, D.; Pei, K.; Fu, Y.; Sun, Z.; Li, S.; Liu, H.; Tang, K.; Han, B.; Tao, Y. Genome-wide analysis of the auxin response factors (ARF) gene family in rice (Oryza sativa). Gene 2007, 394, 13-24. [CrossRef] [PubMed]

74. Chen, Y.; Hao, X.; Cao, J. Small auxin upregulated RNA (SAUR) gene family in maize: Identification, evolution, and its phylogenetic comparison with Arabidopsis, rice, and sorghum: SAUR gene family in maize. J. Integr. Plant Biol. 2014, 56, 133-150. [CrossRef] [PubMed]

75. Feng, S.; Yue, R.; Tao, S.; Yang, Y.; Zhang, L.; Xu, M.; Wang, H.; Shen, C. Genome-wide identification, expression analysis of auxin-responsive GH3 family genes in maize (Zea mays L.) under abiotic stresses: Genome-wide analysis of ZmGH3 gene family. J. Integr. Plant Biol. 2015, 57, 783-795. [CrossRef] [PubMed]

76. Liu, Y.; Jiang, H.; Chen, W.; Qian, Y.; Ma, Q.; Cheng, B.; Zhu, S. Genome-wide analysis of the auxin response factor (ARF) gene family in maize (Zea mays). Plant Growth Regul. 2011, 63, 225-234. [CrossRef] 
77. Wang, Y.; Deng, D.; Bian, Y.; Lv, Y.; Xie, Q. Genome-wide analysis of primary auxin-responsive Aux/IAA gene family in maize (Zea mays. L.). Mol. Biol. Rep. 2010, 37, 3991-4001. [CrossRef]

78. Thines, B.; Katsir, L.; Melotto, M.; Niu, Y.; Mandaokar, A.; Liu, G.; Nomura, K.; He, S.Y.; Howe, G.A.; Browse, J. JAZ repressor proteins are targets of the SCFCOI1 complex during jasmonate signalling. Nature 2007, 448, 661-665. [CrossRef]

79. Yan, Y.; Stolz, S.; Chetelat, A.; Reymond, P.; Pagni, M.; Dubugnon, L.; Farmer, E.E. A downstream mediator in the growth repression limb of the jasmonate pathway. Plant Cell 2007, 19, 2470-2483. [CrossRef]

80. Aida, M.; Beis, D.; Heidstra, R.; Willemsen, V.; Blilou, I.; Galinha, C.; Nussaume, L.; Noh, Y.-S.; Amasino, R.; Scheres, B. The PLETHORA genes mediate patterning of the Arabidopsis root stem cell niche. Cell 2004, 119, 109-120. [CrossRef]

81. Horstman, A.; Willemsen, V.; Boutilier, K.; Heidstra, R. AINTEGUMENTA-LIKE proteins: Hubs in a plethora of networks. Trends Plant Sci. 2014, 19, 146-157. [CrossRef] [PubMed]

82. Jha, P.; Kumar, V. $B A B Y B O O M(B B M)$ : A candidate transcription factor gene in plant biotechnology. Biotechnol. Lett. 2018, 40, 1467-1475. [CrossRef] [PubMed]

(C) 2019 by the authors. Licensee MDPI, Basel, Switzerland. This article is an open access article distributed under the terms and conditions of the Creative Commons Attribution (CC BY) license (http://creativecommons.org/licenses/by/4.0/). 